\title{
Mesoscale Data Assimilation of Myanmar Cyclone Nargis
}

\author{
Masaru KUNII, Yoshinori SHOJI, Mitsuru UENO, Kazuo SAITO \\ Meteorological Research Institute, Tsukuba, Japan
}

(Manuscript received 2 July 2009, in final form 13 November 2009)

\begin{abstract}
A mesoscale data assimilation (DA) system was developed for low latitudes, and DA experiments for the tropical cyclone Nargis were conducted. A tropical cyclone bogus (TCB) procedure was developed for the Bay of Bengal, and the impact was investigated.

The Meso 4D-Var system of the Japan Meteorological Agency (JMA), which was designed for operational mesoscale DA in the mid-latitudes, was modified for DA application in the tropics. Since the relationship of the geostrophic wind was not available near the equator, a weighting function for the regression coefficient matrix for the unbalanced wind was determined based on statistics.

Six DA experiments were performed to produce the initial field at 1200 UTC on April 30, 2008, in order to assess the impact of the DA periods (12 h and $24 \mathrm{~h})$ and two different TCBs. Analyzed initial fields were compared with the JMA global analysis (GANAL), the best track data of RSMC, New Delhi, and the cyclone track estimation of the Indian National Centre for Ocean Information Services (INCOIS).

Precipitable water vapor (PWV) around the cyclone was less than $65 \mathrm{~mm}$ in GANAL; in DA analyses, areas with more than 60-mm PWV were more widespread, especially to the east of the cyclone, and the maximum values exceeded $70 \mathrm{~mm}$ near the cyclone center. The cyclone center position was also improved by DA and the implementation of TCB; however, the cyclonic circulation was underestimated in the 24-h DA without TCB (MA24). Lack of satellite observations of the Bay of Bengal in the first 12-h assimilation windows was determined to be the reason for this underestimation.

Mesoscale numerical predictions using initial fields produced by Meso 4D-Var were conducted, and the results were compared with the prediction using GANAL (control run; GA). Overestimation of the cyclone speed and the resultant positional lag in the control run were ameliorated by modifying the initial field by DA. Differences between the upper flow in the GA analysis and that in DA analyses might account for the difference in cyclone speed. Cyclone development was well predicted except in the MA24 experiment, in which underestimation of cyclonic circulation at the initial time caused subsequent insufficient development. Northward biases were observed in several predicted cyclone tracks. Differences between precipitation in the MA24 and that in other experiments might be one of the reasons for the differences in cyclone track.
\end{abstract}

\section{Introduction}

Tropical cyclones (TCs), including typhoons, are the severest atmospheric phenomena (Yamasaki 1982), and their prediction is one of the most important issues in meteorology. Although the accuracy of the operational NWP at world forecast

Corresponding author: Masaru Kunii, Meteorological Research Institute, 1-1, Nagamine, Tsukuba, Ibaraki, 305-0052, Japan.

E-mail: mkunii@mri-jma.go.jp

(C) 2010, Meteorological Society of Japan centers has improved considerably in recent years, many difficulties still exist in TC prediction. TC track forecasting is one of the most improved aspects of recent NWP; however, statistically there are still $150-200-\mathrm{km}$ errors in $48 \mathrm{~h}$ forecasts, even by the most advanced NWP centers (Komori et al. 2007). The forecast of TC intensity is even more challenging, especially in its early stages. Resolutions and physical processes of current global NWP models are still insufficient to fully resolve and express the evolution of TCs. Another critical point is that most routinely operated mesoscale 
regional data assimilation systems are designed for mid-latitudes. Thus, mesoscale DA study in low latitudes is a key factor in solving this problem.

TC bogus (TCB) data are often necessary over oceans, where enough observational data is not available to determine TCs position and intensity through DA. Based on four-dimensional variational (4D-Var) DA, Zou and Xiao (2000) developed a bogus data assimilation (BDA) scheme and demonstrated its usefulness for the simulation of Hurricane Felix (2000) during its mature stage. Xiao et al. (2000) also investigated the effect of different data in the BDA scheme on the subsequent model forecast. They suggested that the assimilation of sea-level pressure (SLP) was more advantageous than that of the wind component derived from the gradient wind relation. However, $\mathrm{Pu}$ and Braun (2001) and Wu et al. (2006) conducted sensitivity experiments to assess the potential impact of different variables on the BDA scheme, and concluded that the wind field was critical to maintain the TC structure.

On May 2, 2008, cyclone Nargis made landfall in the southwestern part of Myanmar and caused the worst natural disaster in the country, claiming more than one hundred thousand lives (Webster 2008). This cyclone formed in the Bay of Bengal on April 27 and moved eastward, rapidly and intensifying within $24 \mathrm{~h}$ from category-1 to category4 storm on the Saffir-Simpson scale (Simpson 1974). Several studies have been carried out to determine the reason for the rapid development of the cyclone. Lin et al. (2008) investigated the role of a warm anomaly of SST in the Bay of Bengal, and Kikuchi et al. (2009) analyzed the development of the cyclone using satellite-based remote-sensing data.

Cyclones in the Bay of Bengal are generally difficult to analyze because of the insufficiency of available observations. Reale et al. (2009) conducted DA experiments of Nargis using an operational global DA system of the National Aeronautics and Space Administration (NASA). They found that satellite imagery and atmospheric profiles obtained from the Atmospheric Infrared Sounder (AIRS) significantly improved forecasts of the cyclone track and intensity. However, the predicted cyclone central pressure of $998 \mathrm{hPa}$ in their simulation at landfall (1200 UTC on May 02) was weak compared with that in RSMC New Delhi's analysis $(962 \mathrm{hPa})$. One reason for this underestimation seems to be the low horizontal grid resolution in their NWP model $\left(0.5^{\circ} \times 0.67^{\circ}\right)$. Kuroda et al. (2010) and Saito et al. (2010) conducted numerical simulations of Nargis and its associated storm surge using the Japan Meteorological Agency (JMA) nonhydrostatic model (NHM) with a horizontal resolution of $10 \mathrm{~km}$ and the Princeton Ocean Model. They successfully reproduced the storm surge in southern Myanmar; however, the simulated cyclones were $974 \mathrm{hPa}$ without DA.

The main purpose of this study is to analyze accurate initial fields with high resolutions in low latitudes and assess their applicability for forecasting cyclone Nargis. We adopt the mesoscale 4D-Var (Meso 4D-Var) (Ishikawa and Koizumi 2002b; Koizumi et al. 2005) of the JMA, and apply it to the tropics. Since the Meso 4D-Var is designed for operational DA in the mid-latitudes, several modifications were made to use the system in low latitudes. To supplement the shortage of observational data in the Bay of Bengal, pseudoobservations by TCB are prepared using the estimated maximum wind.

Section 2 describes the Meso 4D-Var and its modifications for low latitudes. Section 3 discusses the design of the experiments and the assimilated data. Section 4 presents the results of the experiments, and Section 5 summarizes this study.

\section{Development of a data assimilation system for low latitudes}

\subsection{Meso 4D-Var}

To develop a low-latitude DA system, we modify JMA's Meso 4D-Var and apply it to the tropics. Meso 4D-Var, a four-dimensional DA system developed at JMA, was used as the operational mesoscale DA system from March 2002 to April 2009. ${ }^{1}$ The dynamical core of this system is based on JMA's hydrostatic spectral model (MSM). The system consists of a nonlinear forward model and a simplified adjoint model whose horizontal resolution is $20 \mathrm{~km}$. An incremental approach is employed where the analysis field is made by an outer model with a horizontal resolution of $10 \mathrm{~km}$. Figure 1a presents the analysis domain of JMA's original Meso 4D-Var.

In this study, we shift the domain of the Meso 4D-Var to a low-latitude region that covers the Bay of Bengal (Fig. 1b). Map projection is also

1 Operational mesoscale data-assimilation system was replaced with a nonhydrostatic 4D-Var in April 2009 (Honda and Sawada 2009). 

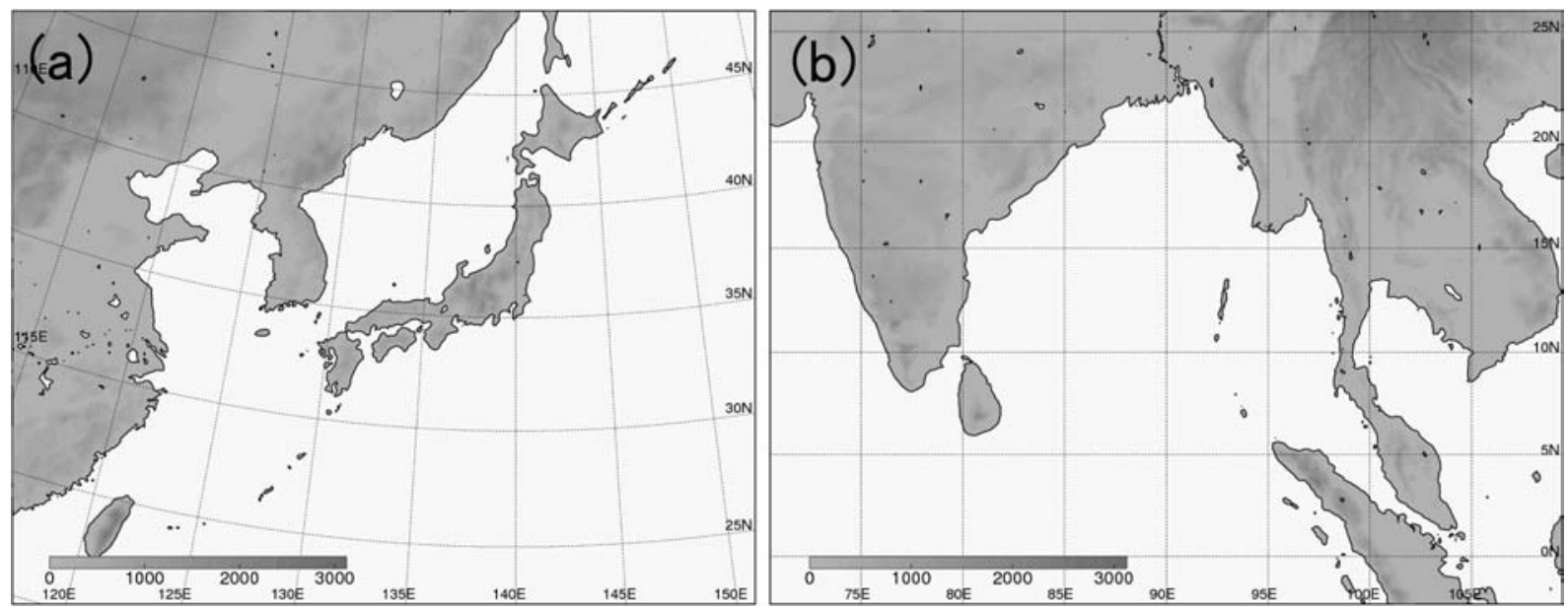

Fig. 1. (a) Domain of JMA Meso 4D-Var system and (b) domain of this study. The original Meso 4D-Var system uses the Lambert conformal projection, while the Mercator projection is used in this study.

changed from the Lambert conformal projection to the Mercator projection for low latitudes. Topography, land-sea distribution, and climatological seasurface temperature data were newly prepared according to the change of domain. Specifications of the new 4D-Var system in the tropics are listed in Table 1.

\subsection{Adjustment of the penalty term}

The cost function of the Meso 4D-Var system is given by

$$
\begin{aligned}
J= & \frac{1}{2}\left(\mathbf{x}_{0}-\mathbf{x}_{0}^{b}\right)^{T} \mathbf{B}^{-1}\left(\mathbf{x}_{0}-\mathbf{x}_{0}^{b}\right) \\
& +\frac{1}{2}\left(\mathbf{x}_{b n d}-\mathbf{x}_{b n d}^{b}\right)^{T} \mathbf{B}^{-1}\left(\mathbf{x}_{b n d}-\mathbf{x}_{b n d}^{b}\right) \\
& +\frac{1}{2}\left(H M \mathbf{x}_{0}-\mathbf{y}^{0}\right)^{T} \mathbf{R}^{-1}\left(H M \mathbf{x}_{0}-\mathbf{y}^{0}\right)+J_{c},
\end{aligned}
$$

where $\mathbf{x}_{0}$ is the model prognostic variables at the initial time, $\mathbf{x}_{\text {bnd }}$ is the lateral boundary condition, $\mathbf{x}_{0}^{b}$ is the first guess of $\mathbf{x}_{0}$, and $\mathbf{x}_{b n d}^{b}$ is the first guesses of $\mathbf{x}_{b n d}, \mathbf{B}$ is the background error covariance matrix, $H$ is the observation operator, $\mathbf{y}^{0}$ is the observation, $\mathbf{R}$ is the observation error covariance matrix, and $M$ represents the nonlinear model. Here, $J_{c}$ is the penalty term that controls the gravity wave noise in the analysis field and is expressed as

$$
J_{c}=\frac{1}{2} r_{j}\left\|\frac{\partial \mathbf{A}}{\partial t}\right\|^{2}
$$

where, $r_{j}$ is the penalty coefficient and $\mathbf{A}$ is the amplitude of the gravity wave mode. Unrealistic growth of gravity waves is suppressed by adding time variation of gravity waves as a constraint condition in the cost function. The coefficient $r_{j}$ is usually determined to keep the $J_{c}$ value constant throughout the minimization process.

In the operational Meso 4D-Var system that targeted the mid-latitudes, $r_{j}=1.2 \times 10^{11}$ was adopted. However, the value of $J_{c}$ tended to vary during the 4D-Var iterations if the original value was used for the tropical domain (Fig. 1b). After some trial and error, we found that a larger value $\left(r_{j}=1.8 \times 10^{11}\right)$ was more appropriate for low latitudes; therefore, we adopted the new value in the following experiments. We confirmed that this adjustment of the penalty coefficient positively impacted both the 4D-Var iterations and the subsequent model forecast.

\subsection{Regression coefficients for balanced wind}

We follow the NMC method (Parrish and Derber 1992) to calculate B. With this method, B is calculated using the statistical differences of errors between the forecast times (FTs) of 6 and $18 \mathrm{~h}$. The number of components of $\mathbf{B}$ in grid space are of the order of $10^{13}$. Therefore, it is impossible to store the covariance in computer memory, and the direct calculation of inverse matrix is impractical. In the Meso 4D-Var system, analysis variables are transformed into control variables that can be regarded as independent of each other. This procedure enables us to calculate the inverse matrix of $\mathbf{B}$.

Meso 4D-Var control variables consist of virtual 
Table 1. Specifications of the original Meso 4D-Var and this study.

\begin{tabular}{|c|c|c|c|}
\hline & \multirow[b]{2}{*}{ Original } & \multirow[b]{2}{*}{ This Study } \\
\hline & & & \\
\hline \multicolumn{2}{|c|}{ Method } & \multicolumn{2}{|c|}{ Incremental method } \\
\hline \multicolumn{2}{|c|}{ Forward model } & \multicolumn{2}{|c|}{$\begin{array}{l}\text { A hydrostatic spectral model with a horizontal resolution of } 10 \mathrm{~km} \text { and } 40 \\
\text { vertical levels up to } 10 \mathrm{hPa} \text {. Three types of precipitation scheme; a large-scale } \\
\text { condensation scheme, a moist convective adjustment scheme for mid-level } \\
\text { convection and a prognostic Arakawa-Schbert scheme for deep cumulus } \\
\text { convection. }\end{array}$} \\
\hline \multicolumn{2}{|c|}{ Adjoint model } & \multicolumn{2}{|c|}{$\begin{array}{l}\text { Same dynamical process as the forward model but has only a few physical } \\
\text { processes: simplified vertical diffusion, simplified long-wave radiation, grid-scale } \\
\text { condensation and moist convection adjustment. }\end{array}$} \\
\hline \multirow{2}{*}{\multicolumn{2}{|c|}{ Lateral boundary condition }} & \multicolumn{2}{|c|}{ Forecast of Global Spectral Model (GSM; TL959L60) } \\
\hline & & $\begin{array}{l}1 \text { hourly original data } \\
\text { (0.1875 degree gausian grid, } 60 \text { model } \\
\text { planes) }\end{array}$ & $\begin{array}{l}6 \text { hourly data distributed from Japan } \\
\text { Meteorological Busuiess Support Center } \\
\text { (JMBSC) } \\
\text { (0.5 degree, } 17 \text { pressure levels) }\end{array}$ \\
\hline \multicolumn{2}{|c|}{ Assimilation window } & 6 hours & 3 hours \\
\hline \multicolumn{2}{|c|}{$\begin{array}{l}\text { Observational data } \\
* \text { All observation data are } \\
\text { treated as observed hourly. } \\
\text { That is all data between }-30 \\
\text { and }+29 \text { minutes to the clock } \\
\text { time are regarded as } \\
\text { observations at the clock time. }\end{array}$} & $\begin{array}{l}\text { Radio-sonde, synop (surface), ship, } \\
\text { buoy, aircraft, wind and PWV fields } \\
\text { retrieved from satellite-based } \\
\text { microwave scatterometer/radiometer, } \\
\text { tropical cyclone bogus. Wind-profiler, } \\
\text { doppler-radar radial wind, Radar- } \\
\text { AMeDAS analyzed rainfall. }\end{array}$ & $\begin{array}{l}\text { Radio-sonde, synop (surface), ship, } \\
\text { buoy, aircraft, wind and PWV fields } \\
\text { retrieved from satellite-based microwave } \\
\text { scatterometer/radiometer, tropical } \\
\text { cyclone bogus (only TCB experiments). }\end{array}$ \\
\hline \multicolumn{2}{|c|}{ Map projection } & Lambert conformal projection & Mercator projection \\
\hline \multirow{2}{*}{$\begin{array}{l}\text { Horizontal grid } \\
\text { resolution (grid } \\
\text { size) }\end{array}$} & Outer model & $\begin{array}{c}10 \mathrm{~km} \\
(361 \times 289)\end{array}$ & $\begin{array}{c}10 \mathrm{~km} \\
(401 \times 301)\end{array}$ \\
\hline & Inner model & $\begin{array}{c}20 \mathrm{~km} \\
(181 \times 145)\end{array}$ & $\begin{array}{c}20 \mathrm{~km} \\
(201 \times 151)\end{array}$ \\
\hline
\end{tabular}

temperature $\left(T_{v}\right)$, model surface pressure $\left(P_{S}\right)$, specific humidity $(q)$, and unbalanced wind $\left(u_{U}, v_{U}\right)$. These variables are regarded as uncorrelated to each other. The relationship between unbalanced and balanced (geostrophic) wind is defined by

$$
\left(\begin{array}{l}
\Delta u_{U} \\
\Delta v_{U}
\end{array}\right)_{k}=\left(\begin{array}{c}
\Delta u \\
\Delta v
\end{array}\right)_{k}-\mathbf{G} \nabla\left(\Delta \Phi+\frac{R_{d} \overline{T_{v}}}{\bar{p}}\right)_{k},
$$

where, $\Phi$ is the geopotential height, $p$ is the atmospheric pressure, $R_{d}$ is the gas constant for dry air, $\overline{T_{v}}$ is the virtual temperature in basic field, and $k$ is the vertical level. Here, $\mathbf{G}$ is the regression coefficient matrix of wind and is expressed as

$$
\mathbf{G}=\left(\begin{array}{ll}
r_{x x} & r_{x y} \\
r_{y x} & r_{y y}
\end{array}\right) \text {. }
$$

The term in parentheses in the second term on the right-hand side of Eq. (3) is called the mass variable.

In the operational Meso 4D-Var, the unbalanced wind is calculated as

$$
\left(\begin{array}{l}
u_{U} \\
v_{U}
\end{array}\right)=\left(\begin{array}{l}
u \\
v
\end{array}\right)-\left(\begin{array}{ll}
r_{x x} & r_{x y} \\
r_{y x} & r_{y y}
\end{array}\right)\left(\begin{array}{l}
u_{g} \\
v_{g}
\end{array}\right),
$$

where, $u_{g}$ and $v_{g}$ are the geostrophic wind components, and $r_{x x}$ is the regression coefficient for components between east-west and east-west, $r_{y y}$ is that for components between north-south and northsouth, $r_{x y}$ is that for components between east-west and north-south, and $r_{y x}$ is that for components between north-south and east-west.

In the operational Meso 4D-Var system, the coefficient matrix $\mathbf{G}$ was determined statistically in the original mid-latitude domain (Fig. 1a). Figure 


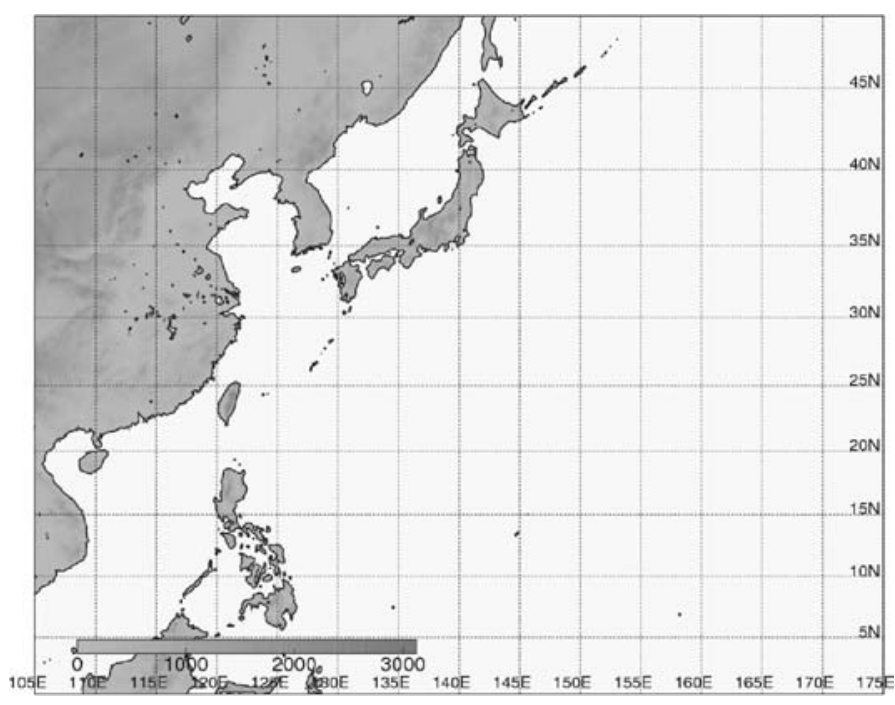

Fig. 2. Domain used to calculate weighting coefficients for the regression coefficient of the geostrophic wind component.

3 presents the regression coefficients in mid-latitude used in the operational Meso 4D-Var as a function of vertical level (Ishikawa and Koizumi 2002a). The geostrophic balance argument has some validity at mid-levels, while it is decreased at low levels due to surface friction.

In this study, it is conceivable that the geostrophic balance argument is not necessarily valid in the new domain since the analysis domain is extended to low latitudes. To solve this problem, we reconstructed $\mathbf{G}$, which was applicable to the tropics. First, we executed preliminary forecast experiments with the domain presented in Fig. 2, in order to investigate the degree of geostrophic balance statistically. The experiments were carried out between August 19-22, 2004 (14 cases). We then divided the whole domain into eight equal parts in the north-south direction and calculated the regression coefficients for each of the eight sub-domains. The result indicated that the geostrophic balance argument was valid in mid-latitudes, but was not satisfied fully near the equator. We determined a new weighting coefficient as a function of latitude based on the amplitudes of coefficients in each partial domain (Fig. 4).

Since the statistical period above seems insufficient for constructing $\mathbf{B}$ itself, we merely utilize the weighting function from the statistical results in order to take into account the latitudinal dependency of the geostrophic balance. We used $\mathbf{B}$ designed for the operational model and multiplied the regression coefficients of mid-latitude by the new weighting coefficient.

\subsection{Tropical Cyclone Bogus (TCB)}

In low latitudes where cyclones are generated, the density of observations is generally sparse. Therefore, in cyclone predictions, especially in the operational NWP, it is common to use pseudodata, called TCB, which expresses typical structures of TCs based on cyclone central pressure and galeforce wind (exceeding $15 \mathrm{~m} / \mathrm{s}$ ) radius.

JMA's operational TCB requires information on the typhoon center position, central pressure, galeforce wind radius, and the surface pressure and geopotential height of the isobaric surface around the typhoon (Onogi 2008).

In the TCB procedure, the radius of the typhoon area $\left(R_{B}(\mathrm{~km})\right)$ is first determined by

$$
R_{B}=\left(1+\frac{2 V_{15}}{f R_{15}}\right)^{1 / 2} R_{15}
$$

where $f$ is the Coriolis parameter, $R_{15}$ the galeforce wind radius, and $V_{15}$ is the specific wind speed of $15 \mathrm{~m} / \mathrm{s}$. Here, $R_{B}$ is equivalent to the radius where the rotation speed around the typhoon center of an air ring initially located at $R_{15}$ becomes zero as it expands outward conserving absolute angular momentum.

The symmetric components of TCB are calculated within $R_{B}$ based on the gale-force wind radius, the central pressure of the typhoon, and the 


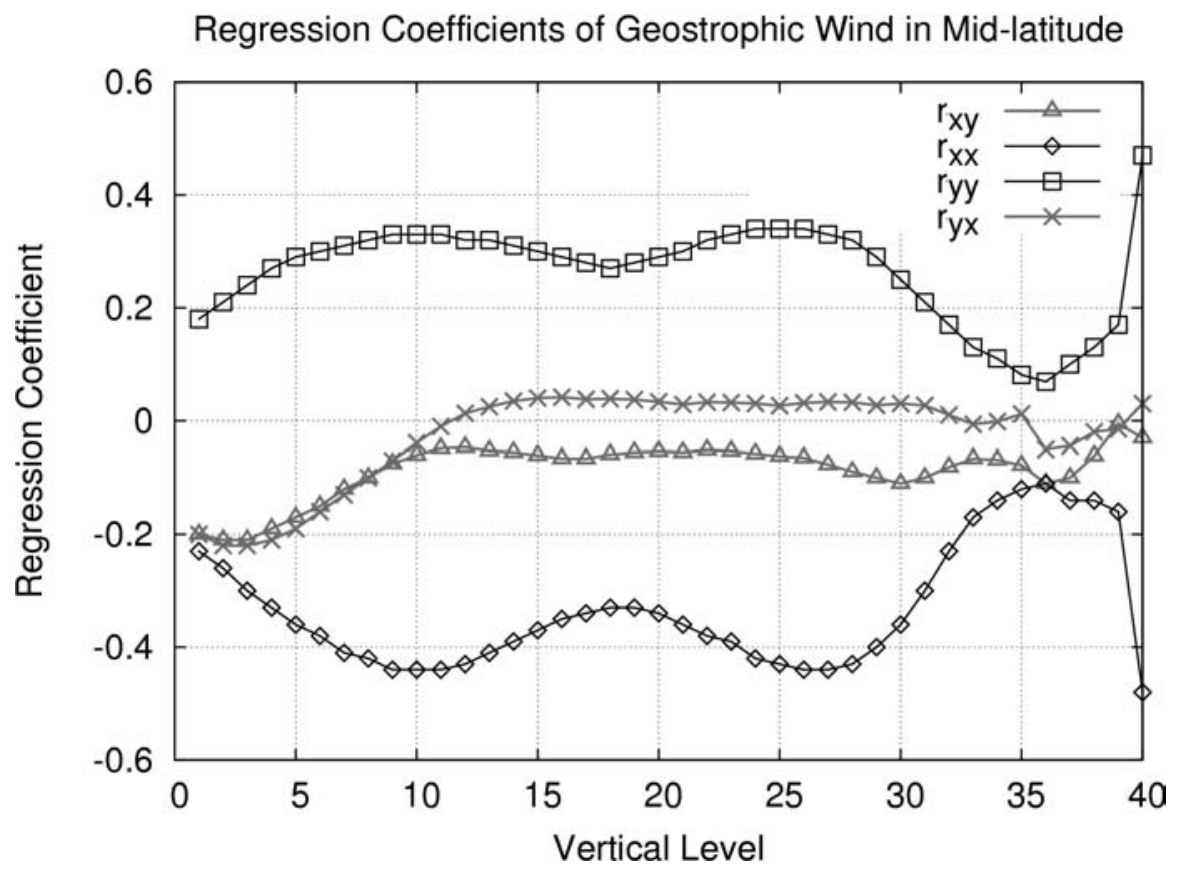

Fig. 3. Regression coefficients of geostrophic wind in mid-latitudes. X-and Y-axis represent the model vertical level and the regression coefficient. Here, $r_{x x}$ (line with open diamonds) represents the regression coefficient for components between east-west and east-west, $r_{y y}$ (line with open square) represents that for components between north-south and north-south, $r_{x y}$ (line with open triangle) represents that for components between east-west and north-south, and $r_{y x}$ (line with $x$ marker) represents that for components between north-south and east-west (Ishikawa and Koizumi 2002a).

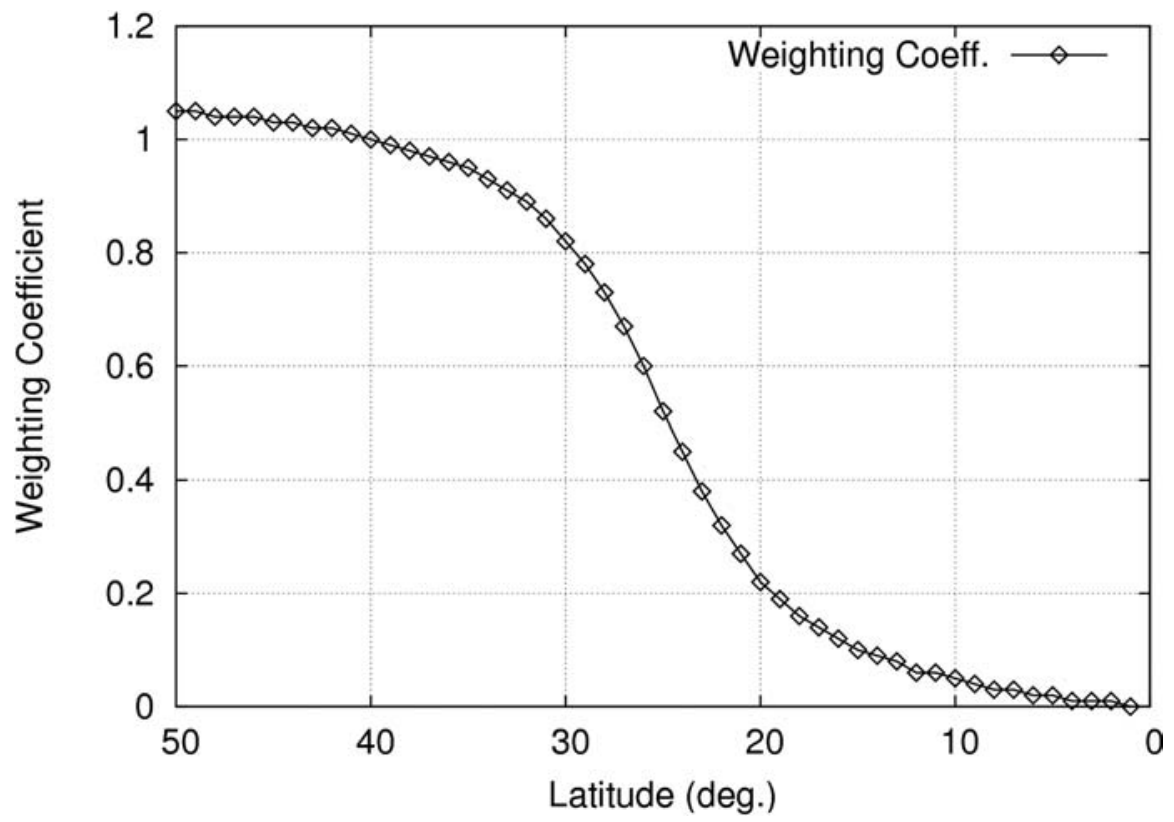

Fig. 4. Weighting coefficients for the regression coefficients of geostrophic wind in mid-latitudes (Fig. 3). 
average values of background SLP, and geopotential height along a circle at $R_{B}$. The symmetric SLP profile is calculated based on the Fujita's (1952) formula as

$$
\begin{aligned}
& P(r)=P_{\max }-\frac{\Delta P}{\left\{1+\left(\frac{r}{R_{0}}\right)^{2}\right\}^{1 / 2}} \\
& \Delta P=P_{\max }-P_{C} \\
& P_{\max }=P_{C}+\frac{P_{B}-P_{C}}{1-\frac{1}{\left\{1+\left(\frac{R_{B}}{R_{0}}\right)^{2}\right\}^{1 / 2}}},
\end{aligned}
$$

where $r(\mathrm{~km})$ is the distance from the center of the typhoon, $P_{C}$ is the central pressure $(\mathrm{hPa}), P_{\max }$ is the pressure $(\mathrm{hPa})$ at infinite radius, and $R_{0}$ is the parameter that determines the SLP profile. When the number of surface synoptic observations within $R_{B}$ exceeds a specified threshold, the SLP profile or $R_{0}$ is modified to fit the observations. The symmetric components of the geopotential height fields are calculated through an analytic function that was developed at JMA to approximate the climatological thermal structure of the tropical cyclone obtained by Frank's (1977) composite study (e.g., see Onogi 2008). After the geopotential height profiles are calculated, the symmetric components of wind fields are derived by assuming gradient wind balance. Near the surface, the wind fields are modified to include the effect of surface friction.

The asymmetric components of TCB are prepared according to Ueno (1995). The asymmetric components of geopotential height and horizontal wind are derived from the background field in the 4D-Var and are added to the symmetric components after the adjustment of the TC center position. One advantage of this method is that it could retain the asymmetric wind components developed in the model such as beta gyres (a pair of counterrotating gyres caused by the advection of planetary vorticity by the typhoon vortex). If the distance between the TC position analyzed by JMA and that represented in the background fields exceeds a threshold $(500 \mathrm{~km})$, the asymmetric components are not considered.

In the operational mesoscale analysis, TCB data created by the above procedure are assimilated as pseudo-observations at the initial time of each assimilation window. The $R_{15}$ and $P_{c}$ of a typhoon in the western North Pacific are determined by
JMA's forecasters every $3 \mathrm{~h}$. However, the JMA estimates of the TC parameters are not routinely available over the Bay of Bengal. To prepare the TCB data for Nargis, we tested the following two TC parameter estimation methods and assessed the impact of resulting TCB data on the forecast.

\section{(a) TCBa}

With this method, Nargis' central pressure $P_{C}$ (or gale-force wind radius $R_{15}$ ) was estimated by using a statistical regression formula between the 10-min maximum wind and central pressure (or gale-force wind radius) obtained from the JMA best-track data from 2004-2007. Here, 10-min maximum winds were determined from 1-min maximum wind analyses provided by the Global Disaster Alert and Coordination System (GDACS). The 1- to 10-min wind conversion was conducted using a conversion factor of 0.871 , a value recommended by the World Meteorological Organization (WMO). The 10-min averaged maximum wind with central pressure and the gale-force wind radius were then fitted to a third-order polynomial.

\section{(b) $\mathrm{TCBb}$}

In the second method, we intended to use the data available for real-time prediction, specifically using the central pressure estimated by the RSMC's best track data for $P_{C}$. Also, instead of resorting to a statistical approach as in the first method, $R_{15}$ was estimated from satellite-based scatterometer (QuikSCAT) sea-wind data. Depending on the availability of QuikSCAT surface-wind observations, $R_{15}$ was first obtained at 1500 UTC on July 28, 1200 UTC on July 29, and 0600 and 1200 UTC on July 30 during the analysis period. A linear interpolation of the values was then made between the corresponding times in order to determine $R_{15}$ every $6 \mathrm{~h}$ over the entire period.

Time sequences of the estimated $P_{C}$ and $R_{15}$ every $6 \mathrm{~h}$ are indicated in Fig. 5, along with the central pressures of the RSMC's best track data and the INCOIS estimation. The gale-force wind radii produced by $\mathrm{TCBb}$ are $1 / 3$ of those produced by TCBa, suggesting that Nargis was an extraordinarily compact tropical cyclone because the regression formula used in TCBa tends to give a typical value of $R_{15}$ for a given maximum wind.

In this study, surface pressure and horizontal wind components at eleven positions around a cyclone were introduced as pseudo-observational data at $6 \mathrm{~h}$ intervals. For wind components, the data at nine levels $(1000,925,850,800,700,600$, 


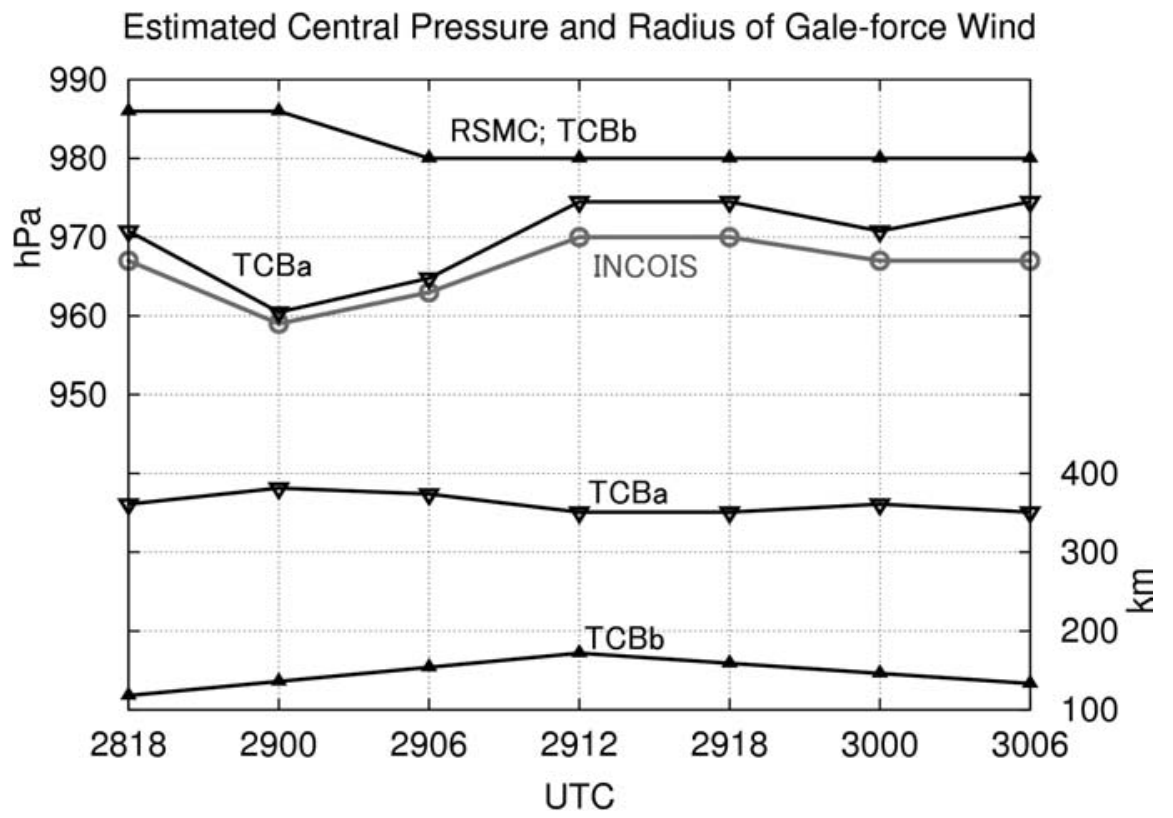

Fig. 5. Sequence of the estimated central pressure (upper half) and the radius of gale-force wind (lower half), along with the cyclone central pressure of the RSMC's best track and the INCOIS's estimation.

500,400 , and $300 \mathrm{hPa}$ ) were extracted from the TCB profiles. These bogus data were applied every $6 \mathrm{~h}$ to the last time slot in the $3 \mathrm{~h}$ assimilation window. To avoid the spin-up problem in the initial fields caused by TCB assimilation, no bogus data were used in the last assimilation window. The observation error and the arrangement of the TCBs were set empirically. Figure 6 presents an example of the alignment of the bogus data at 0600 UTC on April 30.

\section{Experiment design}

We performed numerical experiments using the JMA NHM (Saito et al. 2006; 2007) with a horizontal resolution of $10 \mathrm{~km}$. We focused the initial time in our experiments on 1200 UTC on April 30, 2008, two days before the landfall of Nargis. Numerical simulation and ensemble predictions for Nargis and the associated storm surge were conducted by Kuroda et al. (2010) and Saito et al. (2010) with this initial time. Note that the cyclone's rapid development began after 1200 UTC on May 1. Most of the experiment settings were the same as in the control run of Kuroda et al. (2010) and Saito et al. (2010) except for the initial condition, the model domain (Fig. 1b), and the vertical level configuration (50 stretched levels).
To assess the impact of DA, the following initial conditions were tested.

\section{(1) GA}

JMA's high-resolution global analysis (GANAL) at 1200 UTC April 30.

\section{(2) MA12}

Initial field produced by a successive $12-\mathrm{h} \mathrm{DA}$ using Meso 4D-Var from 0000 UTC to 1200 UTC on April 30, which included four 3-h assimilation windows. Data listed in Table 1 were assimilated with 1-h data slots. In this experiment, TCB was not assimilated to isolate its effect on the TC forecasts compared with the following TCB experiments. The first DA window used GANAL at 0000 UTC on April 30 as the first-guess field. The forecast from the assimilation results of each window were used as the first-guess field of subsequent DA windows. JMA monitors qualities of radio sonde observations by checking their departures from the first-guess field provided by an earlier model forecast. The results are on the JMA's open website (http://qc.kishou.go.jp/). In this study, sonde stations flagged as suspicious in April 2008 were discarded prior to the assimilation.

(3) MA24

Same as the MA12 experiment, except that the 


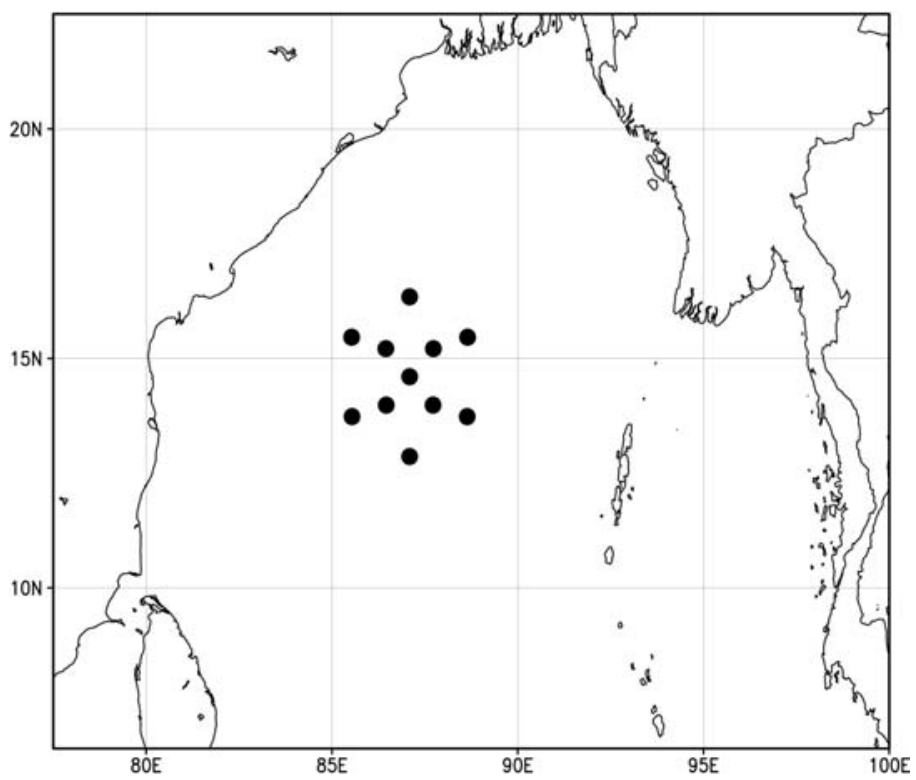

Fig. 6. Alignment of the TCB data at 0600 UTC April 30, 2008.

data assimilation period is of $24 \mathrm{~h}$ from $1200 \mathrm{UTC}$ on April 29 to 1200 UTC on April 30. GANAL at 1200 UTC on April 29 was used as the first guess in the first DA window.

\section{(4) TCBa12}

Same as the MA12 experiment, except that TCBa data were assimilated into the model.

\section{(5) $\mathrm{TCBa} 24$}

Same as the MA24 experiment, except that TCBa data were assimilated into the model.

\section{(6) TCBb12}

Same as the MA12 experiment, except that $\mathrm{TCBb}$ data were assimilated into the model.

\section{(7) $\mathrm{TCBb} 24$}

Same as the MA24 experiment, except that $\mathrm{TCBb}$ data were assimilated into the model.

\section{Results}

\subsection{Differences in initial fields}

Figure 7 indicates the fields of SLP, surface wind, and PWV of GA at 1200 UTC on April 30. Initial

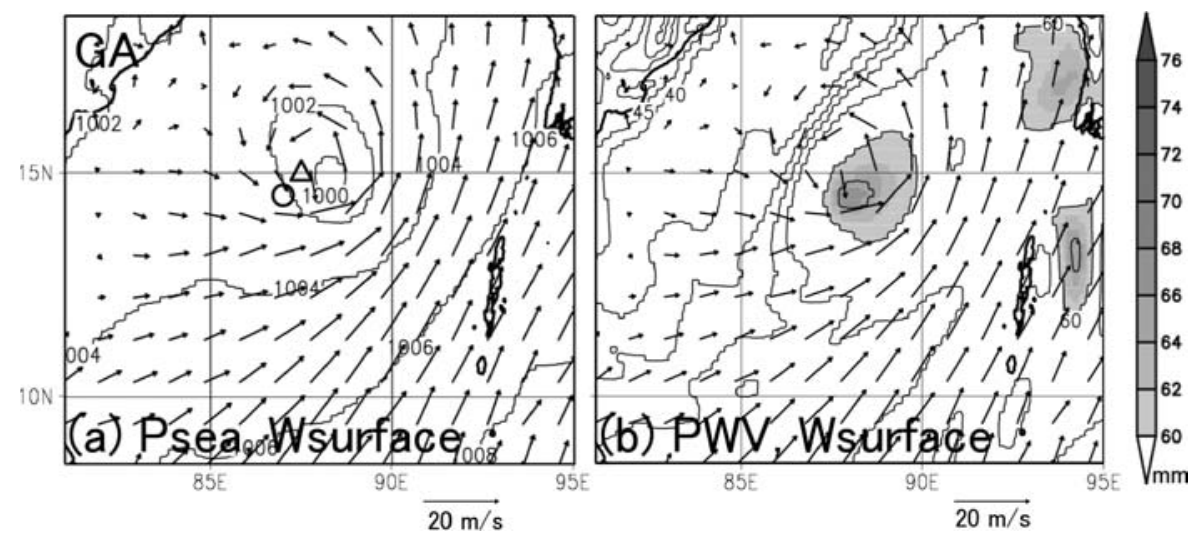

Fig. 7. Distributions of (a) sea level pressure and (b) PWV, along with surface wind field of GA at 12 UTC April 30, 2008. A circle (O) indicates the cyclone center of the RSMC's best track, and a triangle $(\triangle)$ indicates INCOIS's estimation. Contours are drawn at intervals of $2 \mathrm{hPa}$ for sea surface pressure and $5 \mathrm{~mm}$ for PWV. The areas with more than $60 \mathrm{~mm}$ PWV are shaded. 

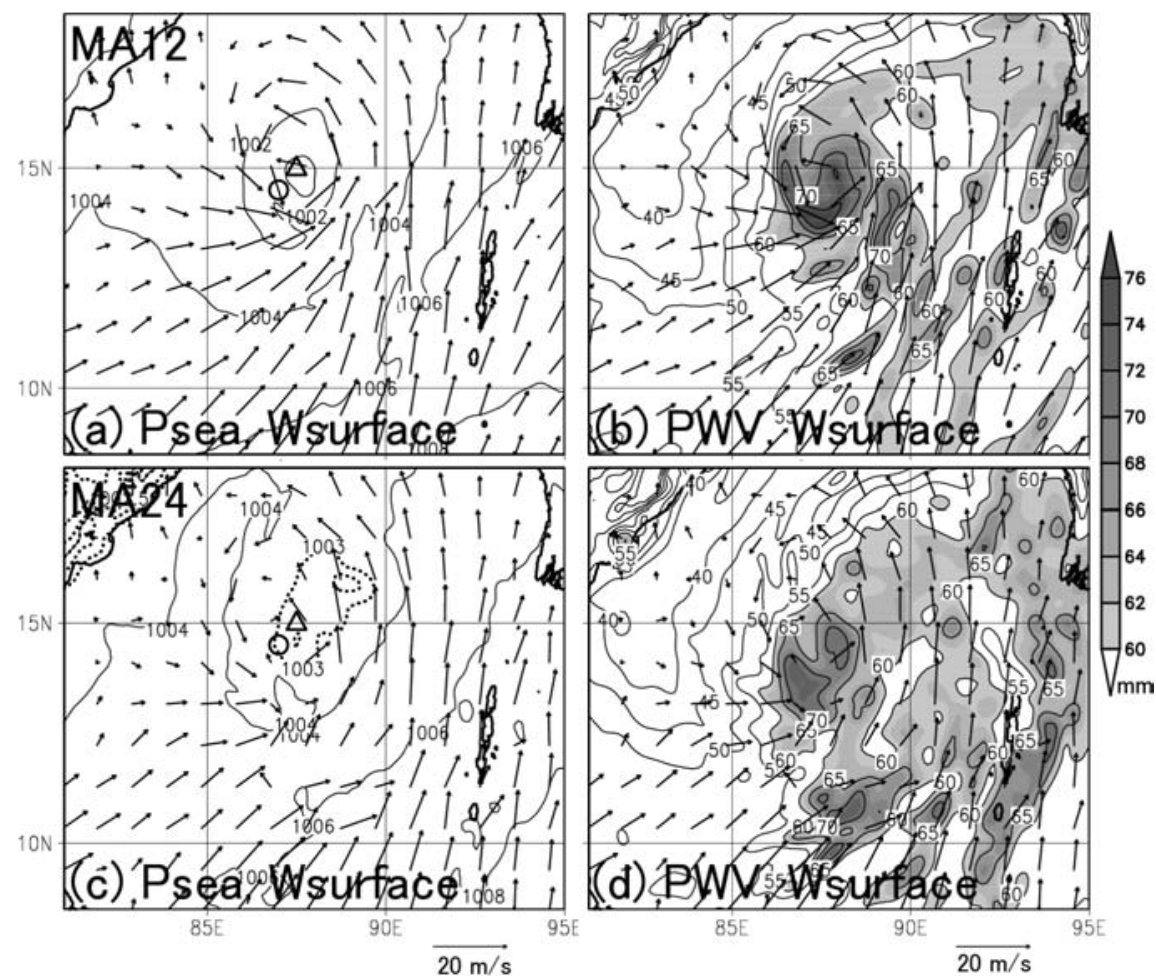

Fig. 8. Same as Fig. 7, but analyzed field by the data assimilation experiments of MA12 (a, b) and MA24 (c, d). Dashed contours in (c) are added to indicate areas of less than $1003 \mathrm{hPa}$ at intervals of $0.5 \mathrm{hPa}$.

fields analyzed by each DA experiment are indicated in Fig. 8 (MA12, MA24), Fig. 9 (TCBa12, TCBa24), and Fig. 10 (TCBb12, TCBb24). Large differences between the PWV fields in GA and other experiments can be seen. In GA, PWV is less than $60 \mathrm{~mm}$ in most of the domain, and the maximum value is $65 \mathrm{~mm}$ near the cyclone center. ${ }^{2}$ In other experiments, areas with more than $60-\mathrm{mm}$ PWV are more widespread, especially to the east of the cyclone, and the maximum values exceed $70 \mathrm{~mm}$ near the cyclone center.

Vertical profiles of relative humidity averaged over the whole domain are presented in Fig. 11. A glance at this figure reveals that the moisture field of GA is drier than those of MA12 and MA24, especially at the $850-300 \mathrm{hPa}$ levels. This is probably a manifestation of the dry bias at the middle and lower levels in the JMA's global spectral model (GSM), which was pointed out by Miyamoto (2009). It seems that DA experiments conducted in this study more appropriately modified the mean

2 In this study, the TC center is defined as the location of minimum SLP. bias of moisture fields that appeared in the first guess. In this case, the assimilation of the PWV and $1 \mathrm{~h}$ accumulated precipitation data retrieved from the satellite-based microwave radiometer effectively ameliorated the analysis fields over the ocean, where observational data were scarce.

Surface-wind fields also indicate certain differences between the GA and other experiments (MA12 and MA24). In GA, southwesterly flow dominates over a wide area southeast of the cyclone (Fig. 7a). In other experiments, southwesterly flows are limited south of the cyclone, and over the sea east of $90^{\circ} \mathrm{E}$, southerly winds prevail (Figs. $8 \mathrm{a}, \mathrm{c})$. These southwesterly and southerly flows converge in the area between the cyclone and the Andaman Islands. This convergence of surface flows in the humid environment mentioned above seems to cause the increase of PWV in the MA12 and MA24 experiments (Figs. 8b, d).

Cyclone central pressures are similar in GA and MA12. However, MA24 results in lower cyclone intensity. Introducing TCB results in a drastic intensification of the cyclone and a decrease of positional errors for the cyclone center (Figs. 9, 10). 


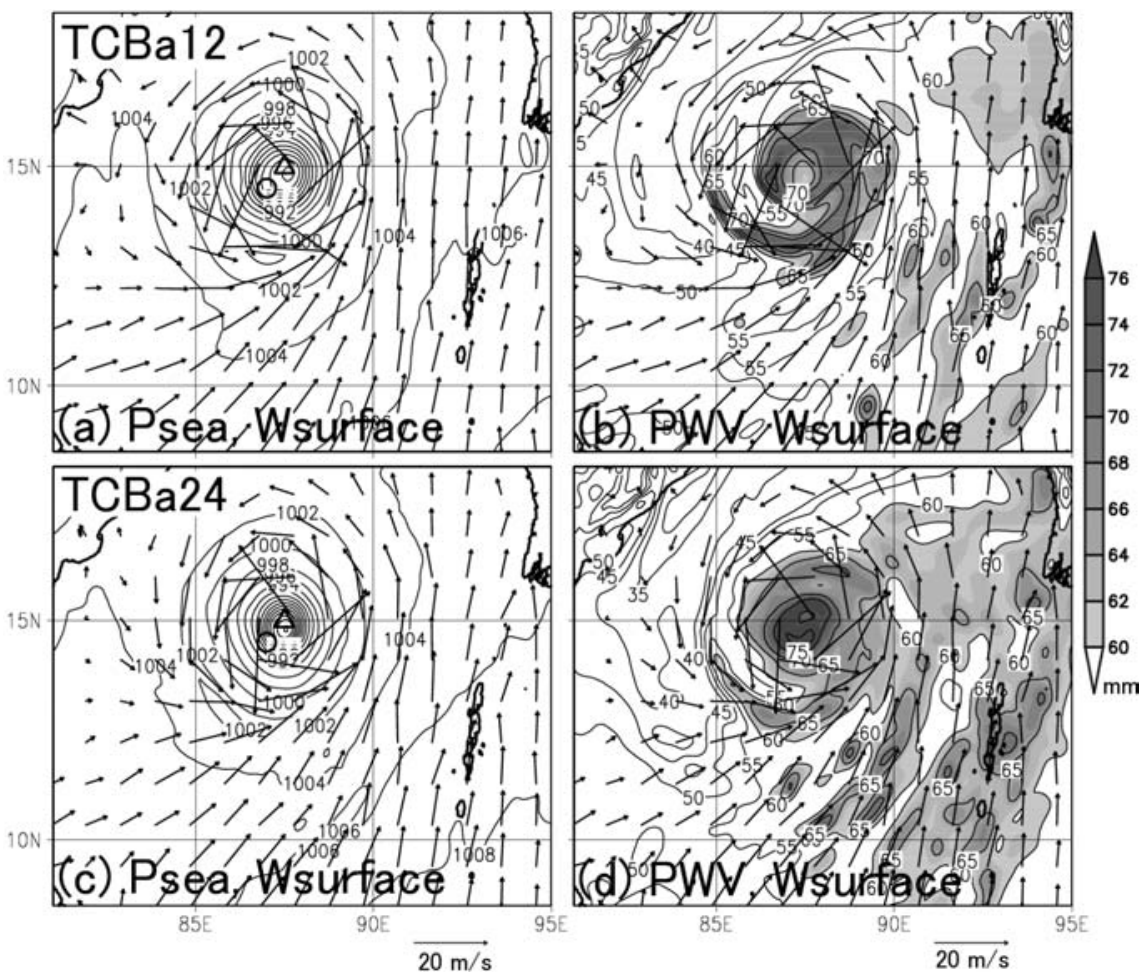

Fig. 9. Same as Fig. 7, but analyzed field by the data assimilation experiments of TCBa12 (a, b) and TCBa24 (c, d).

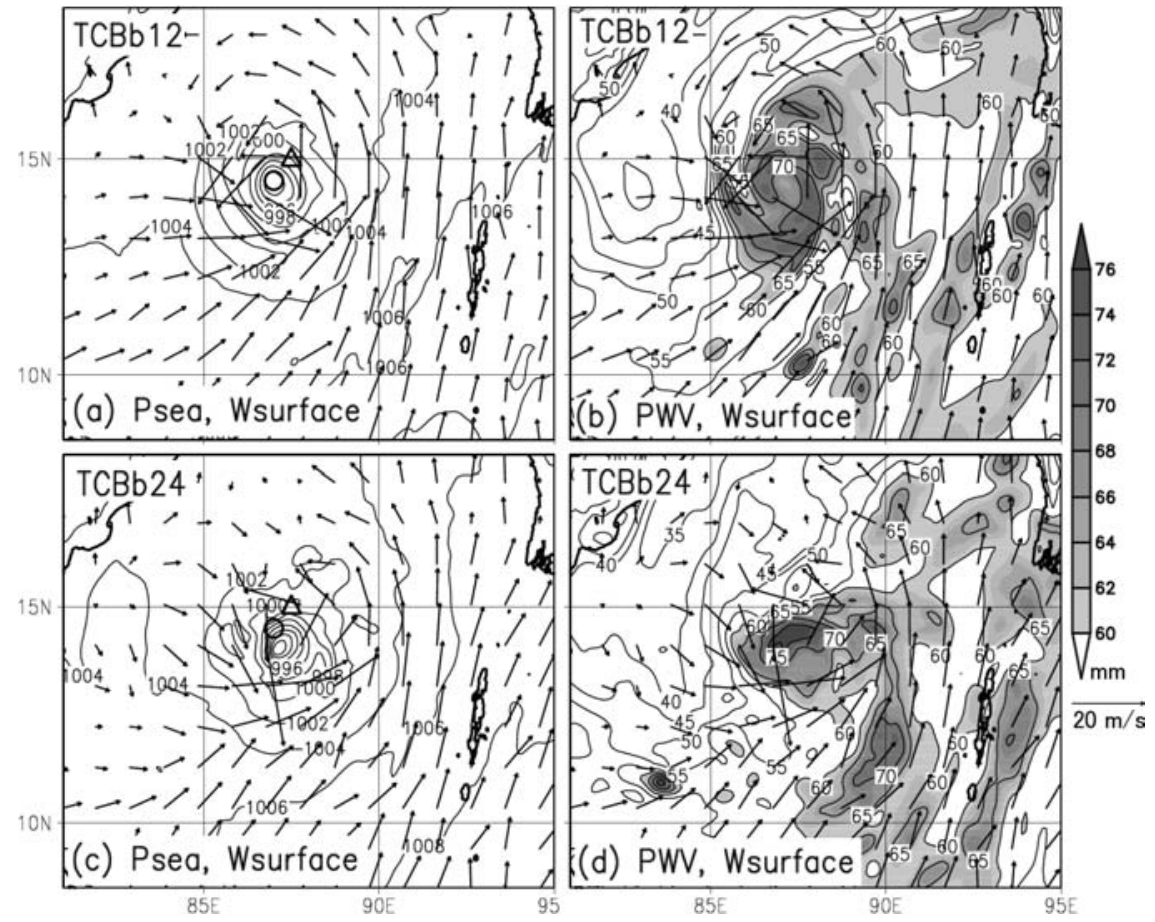

Fig. 10. Same as Fig. 7, but analyzed field by the data assimilation experiments of TCBb12 (a, b) and $\mathrm{TCBb} 24(\mathrm{c}, \mathrm{d})$. 


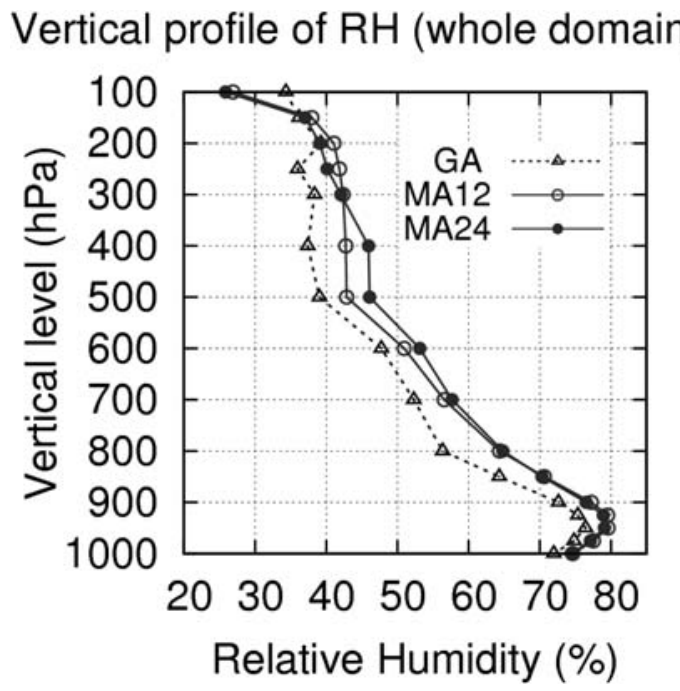

Fig. 11. Vertical distributions of relative humidity averaged over the whole domain for the experiments of GA (dashed line with open triangle), MA12 (gray line with circle), and MA24 (black line with dot).

To determine the reason why MA24 predicted a weaker cyclone, we investigated sea-surface pressure and surface wind from GANAL at 0000 UTC on April 30 (Fig. 12a) and a corresponding 12-h assimilation result in the MA24 experiment (Fig. $12 \mathrm{~b})$. GANAL at this analysis time was used for the first guess of the MA12 experiment. In GA, a cyclonic circulation is observed east of the TC esti- mated by INCOIS; in MA24, horizontal vorticity around the area is relatively weak and deviates northward. This difference probably caused the degradation of the cyclone estimation in MA24.

Figure 13 presents observational data used in each 3-h window in the MA24 experiment. In the first $12 \mathrm{~h}$, no satellite data were available for two assimilation windows (1500-1800 UTC in Fig. 13b and 1800-2100 UTC in Fig. 13c). This lack of data around the cyclone center could cause inadequate analysis of MA24 at 0000 UTC on April 30 (Fig. $12 \mathrm{~b})$. These results suggest the difficulty of cyclone prediction over the sparse-data area.

\section{$4.2 \quad$ Numerical prediction}

Figure 14 presents time series of pressure (Figs. 14a, 14c, and 14e) and track (Figs. 14b, d, f) of the cyclone center predicted by NHM using different initial conditions, along with the best track of RSMC New Delhi and the estimated sequence by the Indian National Centre for Ocean Information Services (INCOIS) (hereafter, collectively called "best tracks").

In the GA experiment, the center pressure became minimum $(969 \mathrm{hPa})$ at $\mathrm{FT}=36(0000 \mathrm{UTC}$ May 2). In best tracks, the center pressures reached minimum values at 0600 UTC on May 2. In this case, the simulated cyclone moved faster than best tracks and made landfall between 0000 and 0600 UTC on May 2 in southern Myanmar. This earlier landfall could be the cause of the earlier occurrence of minimum pressure.

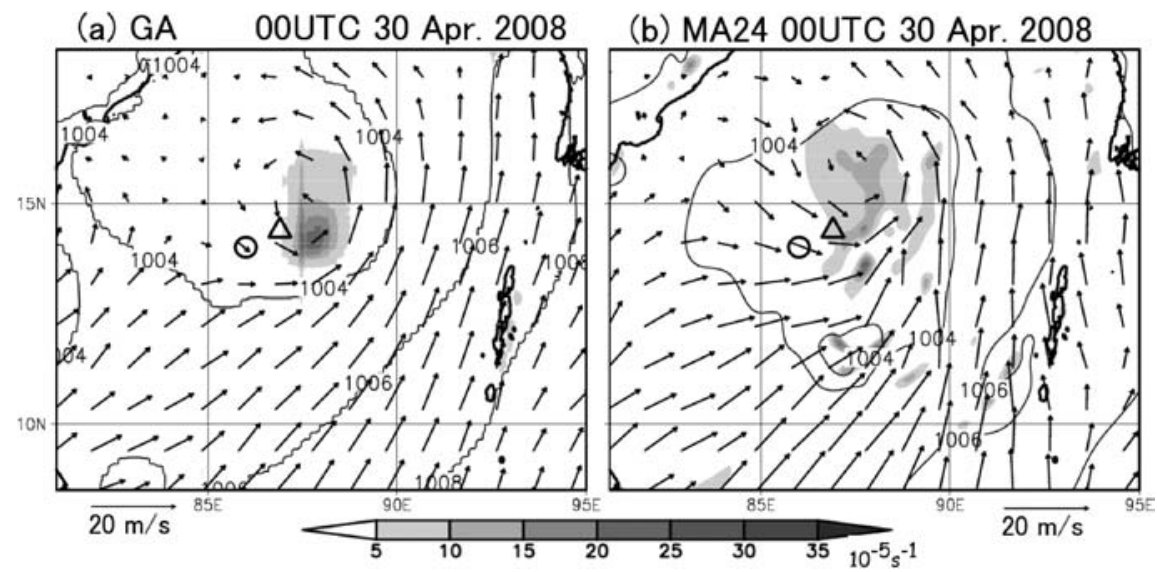

Fig. 12. Fields of SLP and surface wind at 0000 UTC April 30 expressed in GA (a) and MA24 (b). A circle (O) indicates the cyclone centers from the RSMC's best track and a triangle $(\triangle)$ indicates INCOIS's estimation. Contours are drawn at intervals of $2 \mathrm{hPa}$. The areas where the horizontal vorticity exceeds $5 \times 10^{-5} \mathrm{~s}^{-1}$ are hatched. 

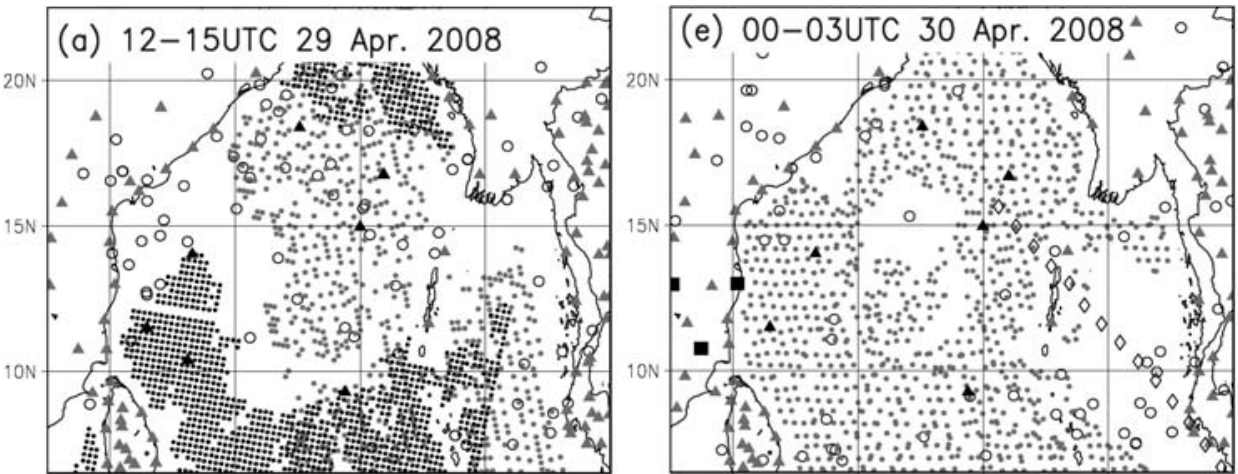

(b) 15-18UTC 29 Apr. 2008
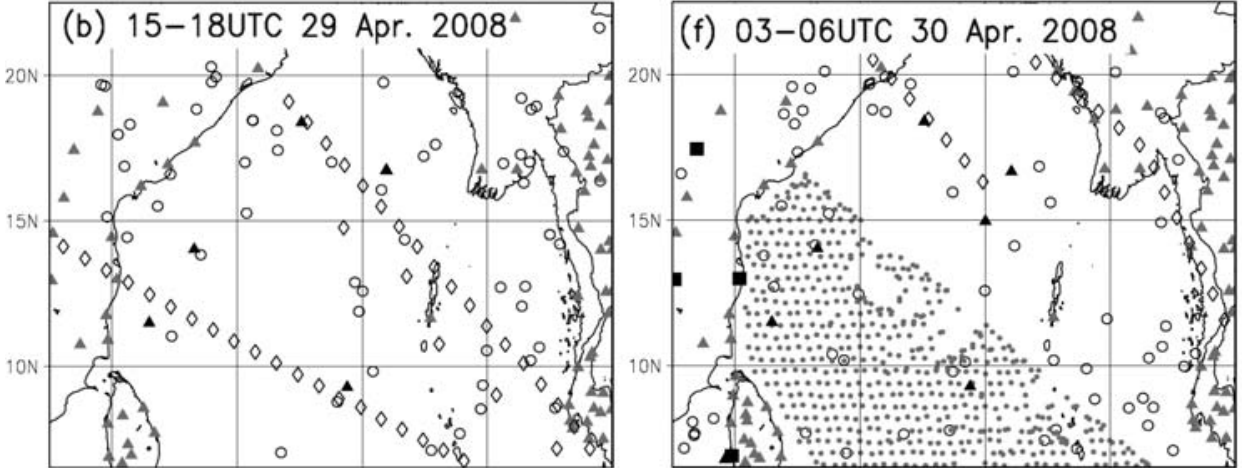

(c) 18-21UTC 29 Apr. 2008
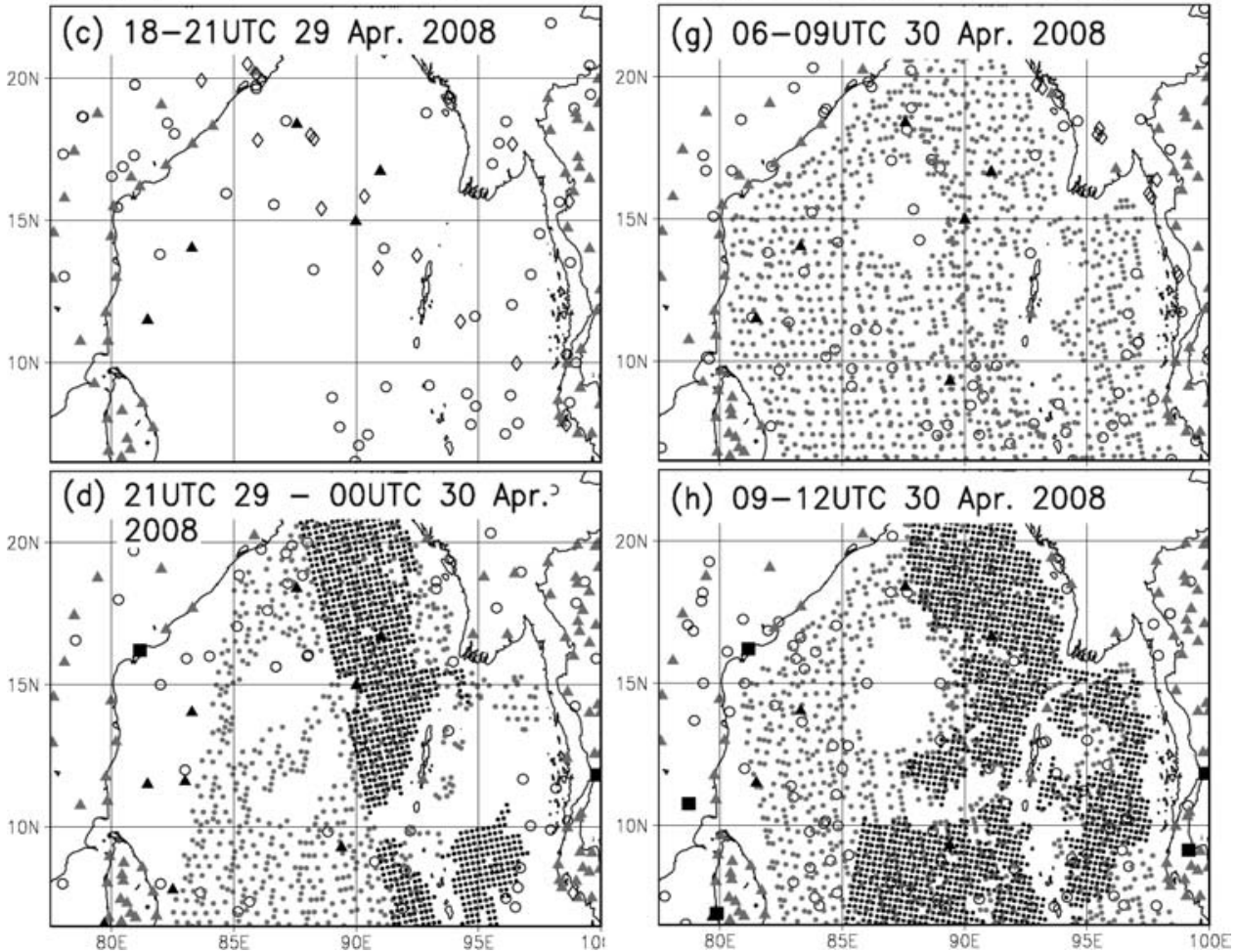

Fig. 13. Distribution of assimilated observational data at each 3-h assimilation window in MA24. Filled square $(\boldsymbol{\square})$ denotes radiosonde, filled gray triangle over land $(\boldsymbol{\Delta})$ indicates surface, filled black triangle over the ocean $(\boldsymbol{\Delta})$ indicates buoy, open diamond $(\diamond)$ indicates aircraft, circle $(\circ)$ indicates satellite obser-

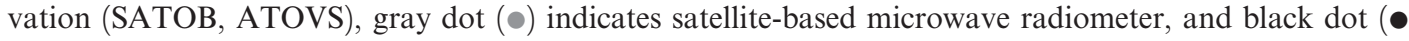
denotes wind field observed by QuikSCAT. 
Central Pressure since 12UTC 30 Apr. 2008
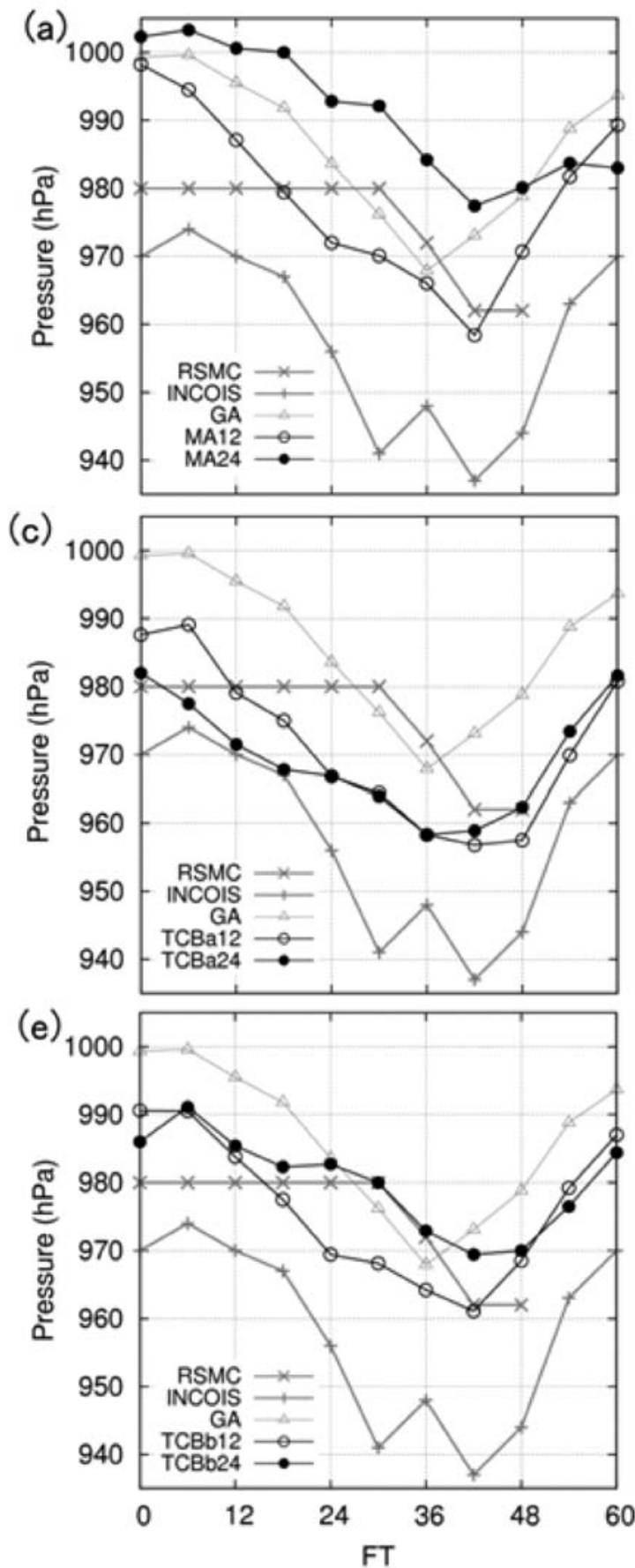

12UTC 30 APR.(FT=0) - 18UTC 02 MAY (FT=54)

(b)
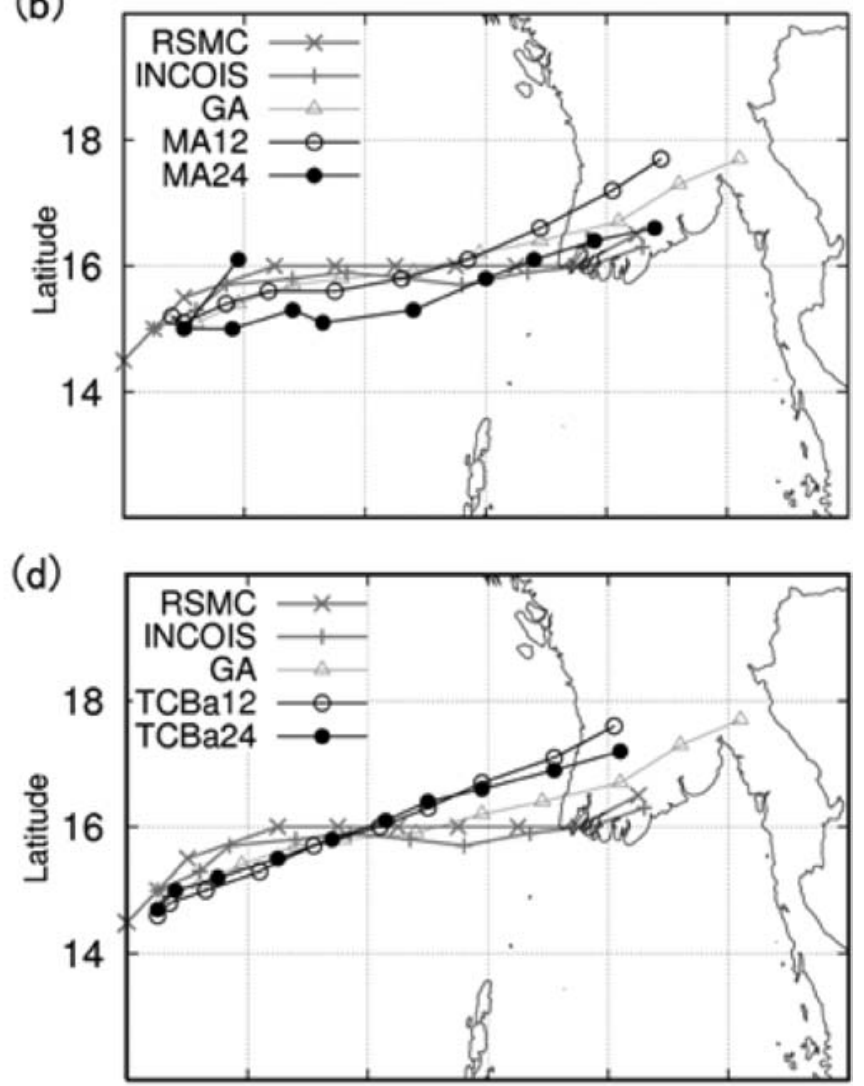

(f)

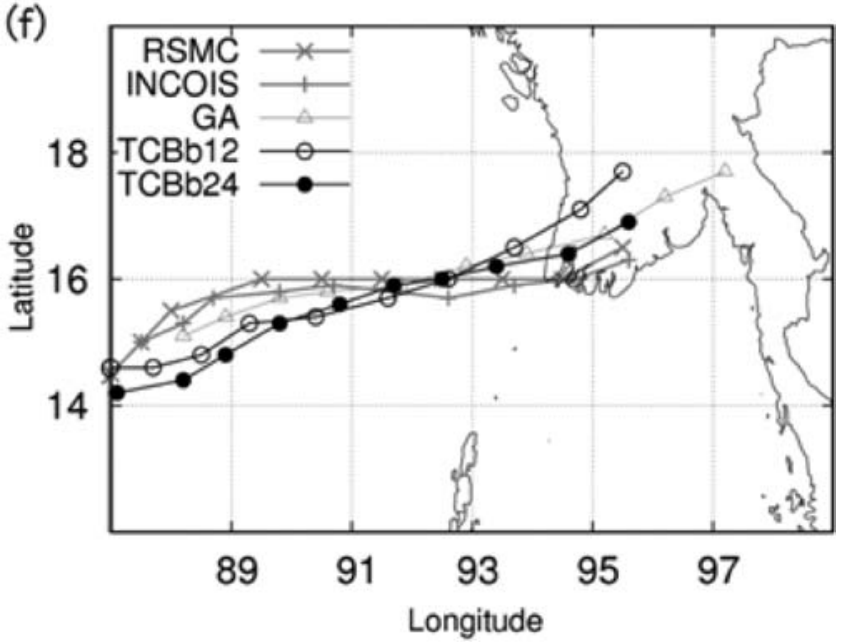

Fig. 14. Time series of pressure (a, c, and e) and track (b, d, and f) of the cyclone center predicted by NHM using different initial fields, along with RSMC New Delhi's best-track sequence (gray line with cross marks) and INCOIS's estimated sequence (gray line with plus marks). 


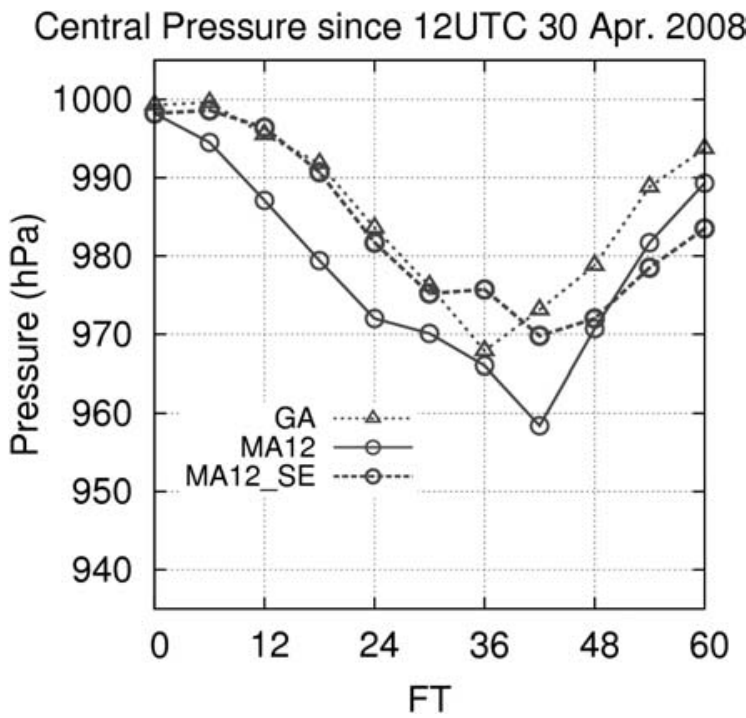

Fig. 15. Same as Fig. 14(a), but forecast results of GA (dashed line with open triangle), MA12 (gray line with circle), and the sensitivity experiment based on MA12 (MA12_SE) (dashed line with circle).

In MA12, the cyclone central pressure at the initial time is almost the same as that in the GA experiment. However, improvements in the prediction of cyclone intensity and speed are obtained. In this experiment, the timing of the minimum pressure is the same as that in the INCOIS analysis, and the cyclone central pressure is consistently deeper than that in the GA experiment. Larger PWV in the analysis (Fig. 8b) may have helped in the cyclone's development.

To investigate the influence of moisture fields on subsequent forecasts, a sensitivity experiment was conducted in which the initial conditions of water vapor were modified. Within a circle of cyclone radius $\left(R_{B}\right.$ in Eq. (8), $\left.450 \mathrm{~km}\right)$ centered in the cyclone, the relative humidity at all levels in the MA12 analysis fields was reduced by $8 \%$. This adjustment can be regarded as the standard deviation of the analysis errors of the relative humidity. After this modification, PWV around the cyclone center reached almost the same magnitude as that of GA. Figure 15 presents the result of this sensitivity experiment. The predicted cyclone intensity weakened, similar to that of GA, especially in the former half of the prediction. Although the initial field of the sensitivity experiment is artificial, we can confirm that the water vapor fields played an important role in the development of the cyclone.
In MA24, the predicted cyclone development was weak. In this case, insufficient analysis of the cyclonic circulation in the initial field (Fig. 8c), as described in the previous subsection, influenced the subsequent prediction. This result suggests that adequate representation of the cyclonic vortex as well as that of the water vapor field in the analysis is essential for successfully simulating the cyclone. Hence, the assimilation of TCB is expected to be beneficial for cyclone forecasting, especially when the initial vortex is not reproduced properly with only the existing observations.

Assimilation of TCB (TCBa12, TCBa24, $\mathrm{TCBb} 12$, and $\mathrm{TCBb} 24)$ intensifies the cyclone. Cyclone central pressures at 1200 UTC April 30 $(\mathrm{FT}=0)$ become deeper compared with experiments without TCB. Predicted cyclone center pressures at the mature stage in the TCBa experiments are between the RSMC's best track and the INCOIS's estimation. The cyclones in the TCBb experiments tend to develop at a slower rate and to a lesser degree than in TCBa. A smaller gale-force wind radius in $\mathrm{TCBb}$ cyclones may have caused this difference. If we assume comparable reliabilities of the two cyclone intensity analyses, the center pressure predicted by TCBa24 seems best.

The positional error at FT $=60$ observed in the GA experiment was reduced in all other experiments (Figs. 14b, d, f). In order to explore the difference in track errors, we calculated the background steering flow, which was defined as a deeplayer mean $(850-300 \mathrm{hPa})$ asymmetric flow within the radius $R_{B}$ centered in the simulated cyclone. Figure 16 indicates its zonal and meridional components at verifying time. The zonal component (Fig. 16a) of GA is almost the largest before landfall $(\mathrm{FT}=42)$. In addition, at the initial time, the cyclone analyzed in GA is located to the east, compared with other experiments. The early landfall of GA is most likely explained by these factors.

As for the meridional components (Fig. 16b), GA has the largest values, especially in the former half of the prediction. The conspicuous northerly bias in the predicted track of GA partly resulted from this overestimation of the meridional flow. However, DA experiments conducted in this study modified the steering flow; consequently, the biases were reduced to some extent. The most notable characteristic in Fig. $16 \mathrm{~b}$ is that the DA experiments with 24-h assimilation time have smaller northward bias than the corresponding experiments with 12-h assimilation time. These results indicate 

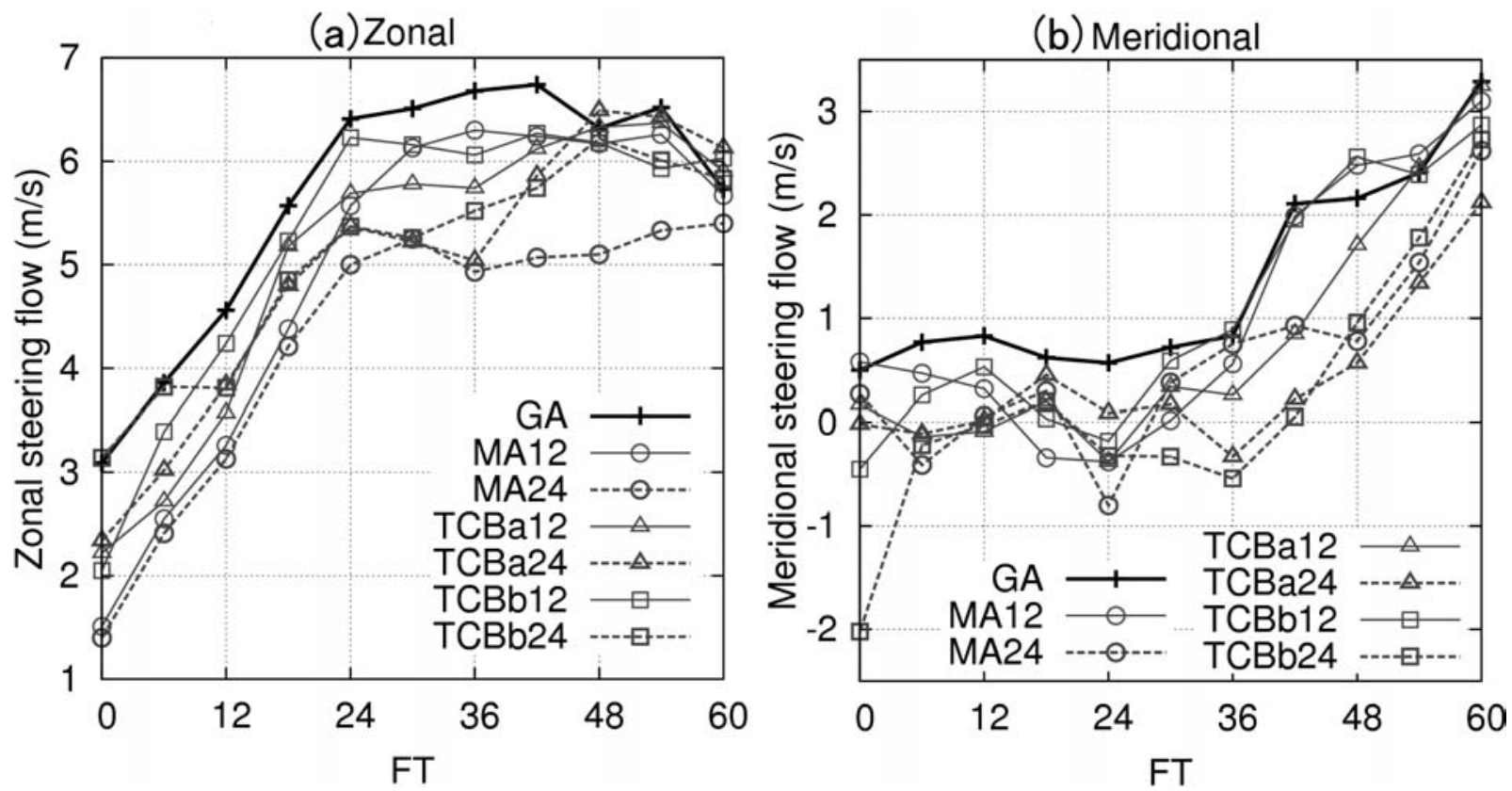

Fig. 16. Steering flow of each experiment: (a) zonal component, (b) meridional component.

that the experiment system in this study worked well and effectively reduced the bias in the assimilation cycles.

Cyclone track predictions in Fig. 14 indicate a southward deflection during the early stage (from $\mathrm{FT}=0$ to $\mathrm{FT}=24)$. However, in the latter half of the predictions, northward deflections were observed in the tracks by TCBa12, TCBa24, TCBb12, and MA12. These northward deflections began at $\mathrm{FT}=36$ in $\mathrm{TCBa} 12$, TCBa24, and $\mathrm{TCBb} 12$, but just before landfall $(\mathrm{FT}=42)$ in MA12. Figure 17 compares accumulated 1-h precipitation amounts at $\mathrm{FT}=36$. In MA24, $\mathrm{TCBb} 12$, and $\mathrm{TCBb} 24$, strong precipitation occurs to the south of the cyclone center, while other experiments except for GA indicate strong precipitation both to the south and north of the cyclone center. In GA, precipitation around the cyclone center is weaker and more symmetrically distributed than in other experiments. Figure 18 presents the precipitation estimated by the Tropical Rainfall Measuring Mission (TRMM) Microwave Imager (TMI). Intense precipitation is seen southwest of the cyclone center, which is comparatively well captured by $\mathrm{TCBb} 24$.

Table 2 summarizes the characteristics of the meridional steering flow and the precipitation pattern around the TC center in each experiment. We speculated that both smaller meridional steering flow and enhanced precipitation (therefore, enhanced mass sink) to the south side of the storm may have contributed to the reduced northward bias in the MA24 and TCBb24 experiments.

However, many possible factors can affect the cyclone track, so further investigation should be conducted in the future.

\section{Summary}

We developed a mesoscale data assimilation system in low latitudes and conducted forecast experiments for the tropical cyclone Nargis. The JMA Meso 4D-Var system, which was designed for operational mesoscale DA in the mid-latitudes, was modified for application to the tropics. The relationship of the balanced (geostrophic) wind was not necessarily appropriate in low latitudes, so a new weight coefficient for the regression coefficient matrix on the unbalanced wind was determined as a function of latitude based on statistics. Additionally, the coefficient for the penalty term that controls gravity waves was made larger.

A procedure for TCB calculation in the Bay of Bengal was developed based on the JMA's operational scheme. At first, we statistically estimated the gale-force wind radius from the 10-min averaged maximum wind to make TCB profiles (TCBa) since 

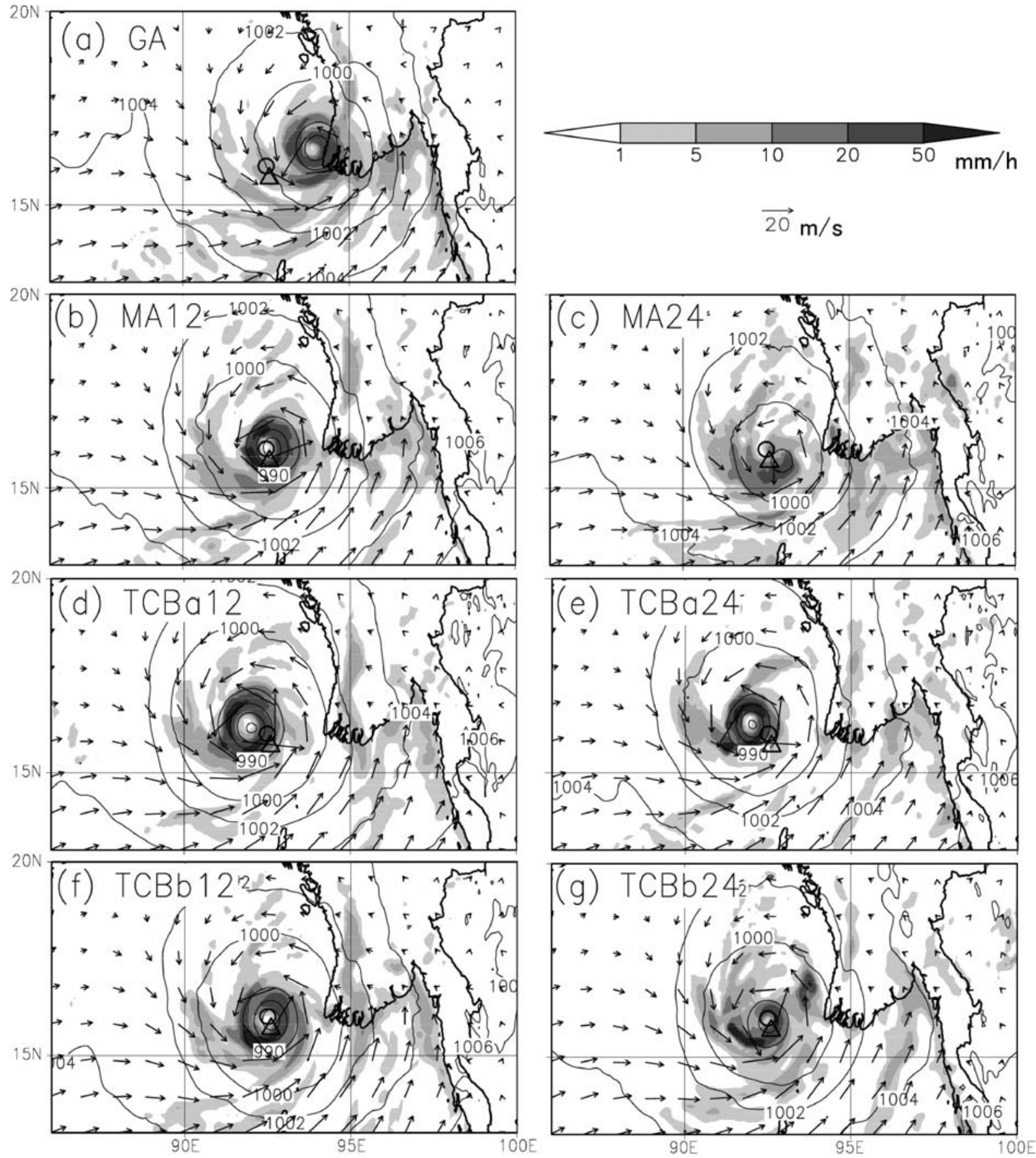

Fig. 17. Predicted SLP, surface wind, and 1-h precipitation amount of the GA experiment at FT $=30$ and 36. A circle $(O)$ indicates the cyclone center of the RSMC New Delhi's best track, and a triangle $(\triangle)$ indicates INCOIS's estimation. Contours larger than $1000 \mathrm{hPa}$ are drawn with intervals of $2 \mathrm{hPa}$ and with intervals of $10 \mathrm{hPa}$ for less than $1000 \mathrm{hPa}$. The areas in which $1-\mathrm{h}$ precipitation amounts exceed $1 \mathrm{~mm}$ are hatched. 


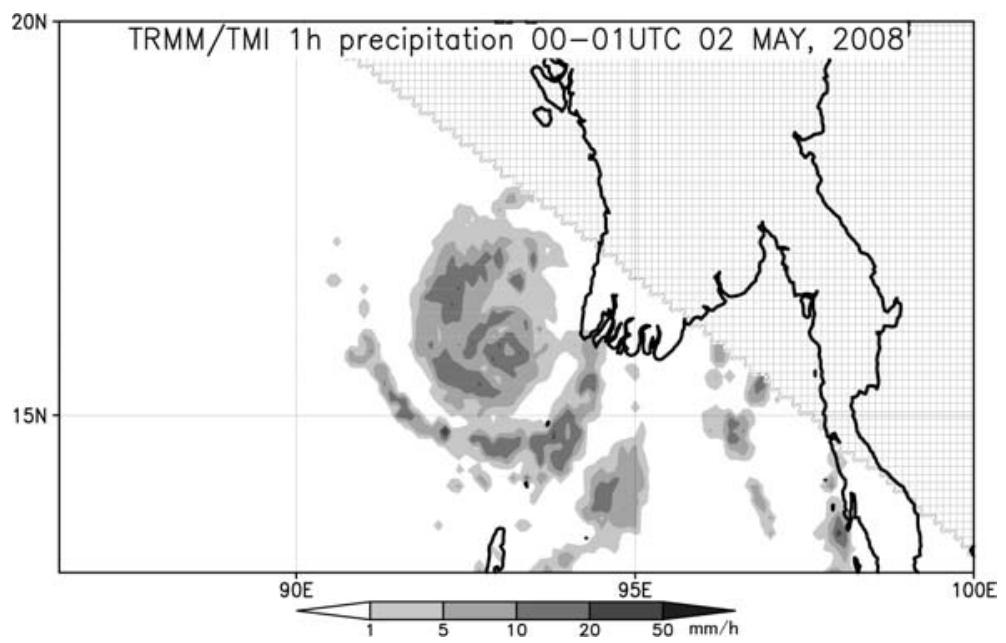

Fig. 18. Observed 1-h accumulated precipitation by TRMM/TMI at 0100 UTC May 2 (interpolated to $0.1^{\circ}$ grids). The observed time corresponds to FT $=37$ of the forecast experiments. Quadrille pattern denotes the area with no data.

Table 2. Characteristics of each experiment: The meridional component of the steering wind (MSTR) (N denotes larger steering flow after FT $=36)$, the precipitation patterns $(\mathrm{PREP})$ around the TC center $(\mathrm{S}$ denotes that strong precipitation occurred south of the TC center), and predicted TC tracks (TRACK) (N denotes that northern bias was observed more clearly).

\begin{tabular}{lccccccc}
\hline & GA & MA12 & MA24 & TCBa12 & TCBa24 & TCBb12 & TCBb24 \\
\hline MSTR & $\mathrm{N}$ & $\mathrm{N}$ & - & - & - & $\mathrm{N}$ & - \\
PREP & - & - & $\mathrm{S}$ & - & - & $\mathrm{S}$ & $\mathrm{S}$ \\
TRACK & $\mathrm{N}$ & $\mathrm{N}$ & - & $\mathrm{N}$ & $\mathrm{N}$ & - \\
\hline
\end{tabular}

no information about the gale-force wind radius was available for Nargis.

Cyclone intensity forecasts were improved except in the MA24 experiment. In MA24, insufficient analysis of the cyclonic circulation at the initial time yielded an underestimation of cyclone intensity in the subsequent prediction with NHM. Lack of satellite observations in the Bay of Bengal in the assimilation windows in MA24 caused deterioration of the analysis field.

Through the assimilation, large differences were found in the initial field of each experiment. In GA, the PWV was less than $60 \mathrm{~mm}$ in most of the domain; however, in other experiments, areas with more than 60-mm PWV were more widespread, especially east of the cyclone and maximum values exceeding $70 \mathrm{~mm}$ near the cyclone center. Overestimation of the cyclone speed in the GA (control) experiment was ameliorated in the data assimilation experiments. Predicted cyclones indicated north- ward biases in the tracks, compared with the best track data. Considering that the northward displacement of the tropical cyclone vortex by the beta effect (Chan and Williams 1987) is generally proportional to the initial strength of the outer flow (e.g., Fiorino and Elsberry 1989), the overestimation of gale-force wind radius might be a contributing factor in the northward track deflection in TCBa. We checked this conjecture by verifying the gale-force wind radii used for the TCBa analyses against those estimated from the QuikSCAT seasurface winds. Verification indicated that the statistically estimated gale-force wind radii were considerably overestimated (i.e., larger bogus vortices were represented in the TCBa analysis fields), suggesting a significant contribution of ill-defined initial vortices in TCBa to the northward track bias.

For better simulation, we introduced a new TCB $(\mathrm{TCBb})$, which used the gale-force wind radius analyzed from QuikSCAT wind data and the RSMC's 
best track data available for operational purposes. As a result, the northward bias in the storm track was reduced, especially in the $\mathrm{TCBb} 24$ experiment. This result suggests the importance of bogus vortex size and intensity in predicting the cyclone track.

No regional DA system suitable for TC forecast in the tropics has been developed and used in previous studies. Although the horizontal resolution of our DA system in the present study is not necessarily adequate to express the fine structure of the cyclone's eye, the system can provide more appropriate initial fields in low latitudes for the mesoscale cyclone forecast. Moreover, since the RSMC besttrack data and QuikSCAT wind data required in the TCB process can be obtained in near real time, it would be possible to operate the DA system and provide forecast information in a timely manner. It is expected that in the future such DA systems will contribute to mitigating meteorological disasters in the low-latitude region, including Southeast Asia.

More reliable procedures on TCB methods (e.g., observation error settings and alignment) in the Bay of Bengal should be investigated further. Retrieval of PWV data from ground-based GPS stations around the Bay of Bengal has been conducted by the authors' group. Results of the DA experiment will be given as part II of our study.

\section{Acknowledgments}

We are grateful to two anonymous referees for their valuable comments, which significantly improved the manuscript.

This work was partly supported by the MEXT Special Coordination Funds for Promoting Science and Technology International Research for Prevention and Mitigation of Meteorological Disasters in Southeast Asia Grant-in-Aid for Scientific Research, represented by Professor Shigeo Yoden of Kyoto University. The authors are grateful to Masami Tokuno and Shunsuke Hoshino of MRI for providing us QuikSCAT and TRMM analysis data, valuable comments, and information.

\section{References}

Chan, J. C.-L., and R. T. Williams, 1987: Analytical and numerical studies of the beta-effect in tropical cyclone motion. Part I: Zero mean flow. J. Atmos. Sci., 44, 1257-1265.

Fiorino, M., and R. L. Elsberry, 1989: Some aspects of vortex structure related to tropical cyclone motion. J. Atmos. Sci., 46, 975-990.

Frank, W. M., 1977: The structure and energetics of the tropical cyclone I. Storm structure. Mon. Wea. Rev., 105, 1119-1135.

Fujita, T. 1952: Pressure distribution within Typhoon. Geophys. Mag., 23, 437-451.

Honda, Y., and K. Sawada, 2009: Upgrade of the Operational Mesoscale 4D-Var at the Japan meteorological Agency. CAS/JSC WGNE Res. Activ. Atmos. Oceanic Model, 1.11-1.12.

Ishikawa, Y., and K. Koizumi, 2002a: Mesoscale 4dimensional variational data assimilation. Annual report of Numerical Prediction Division, 48, 37-59. (in Japanese)

Ishikawa, Y., and K. Koizumi, 2002b: One month cycle experiments of JMA mesoscale 4-dimensional variational data assimilation (4D-Var) system. Research Activities in Atmospheric and Oceanic Modeling. 32, WMO/TD-No. 1105,01.26-01.27.

Kikuchi K., B. Wang, H. Fudeyasu (2009), Genesis of tropical cyclone Nargis revealed by multiple satellite observations, Geophys. Res. Lett., 36, L06811, doi:10.1029/2009GL037296.

Koizumi, K., Y. Ishikawa, and T. Tsuyuki, 2005: Assimilation of Precipitation Data to the JMA Mesoscale Model with a Four-dimensional Variational Method and its Impact on Precipitation Forecasts. SOLA, 1, 45-48.

Komori, T., M. Yamaguchi, R. Sakai, and Y. Takeuchi, 2007: WGNE Intercomparison of tropical cyclone forecasts with operational global models: Quindecennial Report. Science Highlights, WCRP. (Available online at http://wcrp.wmo .int/documents/WGNE_TC_Intercomparison _Quindecennial_Quicklook15Anniversary.pdf)

Kuroda, T., K. Saito, M. Kunii, and N. Kohno, 2010: Numerical Simulation of Myanmar Cyclone Nargis Part I: Forecast Experiment with NHM and Simulation of Storm Surge. J. Meteor. Soc. Japan, 88, 521-545.

Lin, I.-I., C.-H. Chen, I.-F. Pun, W. T. Liu, and C.-C. Wu, 2009: Warm ocean anomaly, air sea fluxes, and the rapid intensification of tropical cyclone Nargis, Geophys. Res. Lett., 36, L03817, doi:10.1029/2008GL035815.

Miyamoto, K., 2009: Dry bias in the middle troposphere of the GSM. Annual report of Numerical Prediction Division, 55, 68-70. (in Japanese)

Onogi, K., 2008: Typhoon Bogus, Meterological Research Note, 217, 120-127. (in Japanese).

Parrish, D. F., and J. C. Derber, 1992: The National Meteorological Center's Spectral StatisticalInterpolation Analysis System, Mon. Wea. Rev., 120, 1747-1763.

Pu, Z.-X., and S. A. Braun, 2001: Evaluation of bogus vortex techniques with four-dimensional variational data assimilation. Mon. Wea. Rev., 129 , 2023-2039.

Reale, O., W. K. Lau, J. Susskind, E. Brin, E. Liu, L. P. 
Riishojgaard, M. Fuentes, and R. Rosenberg, AIRS impact on the analysis and forecast track of tropical cyclone Nargis in a global data assimilation and forecasting system, G. R. L., 36, L06812, doi:10.1029/2008GL037122.

Saito, K., T. Fujita, Y. Yamada, J. Ishida, Y. Kumagai, K. Aranami, S. Ohmori, R. Nagasawa, S. Kumagai, C. Muroi, T. Kato, H. Eito, and Y. Yamazaki, 2006: The operational JMA Nonhydrostatic Mesoscale Model. Mon. Wea. Rev., 134, 1266-1298.

Saito, K., J. Ishida, K. Aranami, T. Hara, T. Segawa, M. Narita, and Y. Honda, 2007: Nonhydrostatic atmospheric models and operational development at JMA. J. Meteor. Soc. Japan, 85B, 271-304.

Saito, K., T. Kuroda, M. Kunii, and N. Kohno, 2010: Numerical Simulation of Myanmar Cyclone Nargis Part 2: Ensemble prediction. J. Meteor. Soc. Japan, 88, 547-570.

Simpson, R. H., 1974: The hurricane disaster potential scale. Weatherwise, 27, 169-186.

Ueno, M. 1995: A study on the impact of asymmetric components around tropical cyclone center on the accuracy of bogus data and the track forecast. Meteorol. Atmos. Phys., 56, 125-134.

Webster, P. J., 2008: Myanmer's deadly daffodil. Nature Geoscience doi: 10.1038/ngeo257.

Wu, C.-C., K.-H. Chou, Y. Wang, and Y.-H. Kuo, 2006: Tropical cyclone initialization and prediction based on four-dimensional variational data assimilation. J. Atmos. Sci., 63, 2383-2395.

Xiao, Q., X. Zou, and B. Wang, 2000: Initialization and simulation of a landfalling hurricane using a variational bogus data assimilation scheme. Mon. Wea. Rev., 128, 2252-2269.

Yamaguchi, M., R. Sakai, M. Kyoda, T. Komori, and T. Kadowaki, 2009: Typhoon Ensemble Prediction System developed at the Japan Meteorological Agency. Mon. Wea. Rev., 137, 2592-2604.

Yamasaki, M., Typhoon. Tokyodo Pub., 206pp (in Japanese).

Zou, X., and Q. Xiao, 2000: Studies on the initialization and simulation of a mature hurricane using a variational bogus data assimilation scheme. J. Atmos. Sci., 57, 836-860. 